\section{Properties and Performance of Extended Target Motion} Analysis

\author{
J. P. LE CADRE, Member, IEEE \\ O. TRÉMOIS \\ IRISA/CNRS \\ France
}

The problem of target motion analysis (TMA) has been the subject of an important literature. However, present methods use data estimated by a short time analysis (azimuths, Dopplers, etc.). For far sources, the nonstationarities of the array processing outputs, induced by the sources motion may be simply modeled. This model leads to consider directly a spatio-temporal TMA. Then new (spatio-temporal) data can be estimated. These estimates correspond to a long time analysis. Further, note that they are estimated independently of the (classical) bearings. In this general framework, the concept of source trajectory replaces the classical instantaneous bearings. Corresponding TMA algorithms are then studied. Then the study of statistical performance is carefully studied.

Manuscript received May 10, 1993; revised September 13, 1994.

IEEE Log No. T-AES/32/1/00766.

This work was supported by DCN/Ingénierie (Direction Constructions Navales), France.

Authors' current addresses: J. P. Le Cadre, IRISA/CNRS, Campus de Beaulieu, 35042 Rennes Cedex, France; O. Trémois, Thomson-Sintra DASM, Centre de Brest, Route de Sainte-Anne du Portzic, 29601 Brest, France.

$\overline{0018-9251 / 96 / \$ 10.00 ~(c) ~} 1996$ IEEE

\section{INTRODUCTION}

Conceptually, the basic problem in target motion analysis (TMA for the sequel) is to estimate the trajectory of an object (i.e., position and velocity) from noise-corrupted sensor data [1]. This work is mainly devoted to passive sonar applications and, therefore, no a priori knowledge about sources location is available. In the whole sonar processing, the TMA step corresponds to a high-level processing. It uses basically the estimated bearings, themselves obtained from array processing, tracking, data association, etc.

The performance of any TMA algorithm is conditioned by the statistical quality (i.e., bias and variance) of the estimated bearings. The variance of the estimated bearings are themselves conditioned by the integration time in the basic array processing. This is also true for source tracking and data association [2]. For a fixed source, the statistical quality of the estimated bearings can be improved by increasing the integration time [3]. However, this is not at all true for a moving source [4], since, in this case, the integration time is limited by the nonstationary nature of the problem. More precisely $[4,5]$ it has been shown that for a given target-observer encounter an optimal integration time exists and may be analytically calculated. However, the practical interest of such calculation is limited by the basic hypothesis: the source trajectory is assumed to be known.

Classical array processings correspond to a short time analysis and, thus, its performance is basically limited by the nonstationary nature of the problem. The natural way to overcome this problem consists in considering a true spatio-temporal analysis instead of classical array processing. More precisely, a model of the nonstationary spatio-temporal data may be easily derived. A moving source is then parameterized by a (simplified) spatio-temporal model. This model is defined by two (or more) parameters, its validity (in time) is also limited but is greatly enhanced with respect to (WRT) the classical one. Thus, it corresponds to a long time analysis. The estimation of these spatio-temporal source models has been previously considered and simple and efficient methods have been derived $[6,7]$ for that aim. The scope of this work is restricted to TMA applications. For that purpose, new (spatio-temporal) data will be included in the TMA algorithms. This amounts to replace the notion of (instantaneous) bearing by that of source trajectory in the TMA data.

Let us present now the general organization of this work. In Section II, a simplified model of the source motion (seen by the array) is considered, its relations with classical parameters of TMA (source state vector) are derived. Thus a new parameter is introduced, named $\dot{k}$ it represents the spatial frequency rate. It is stressed that it can be estimated independently of the corresponding spatial frequency. The statistical 


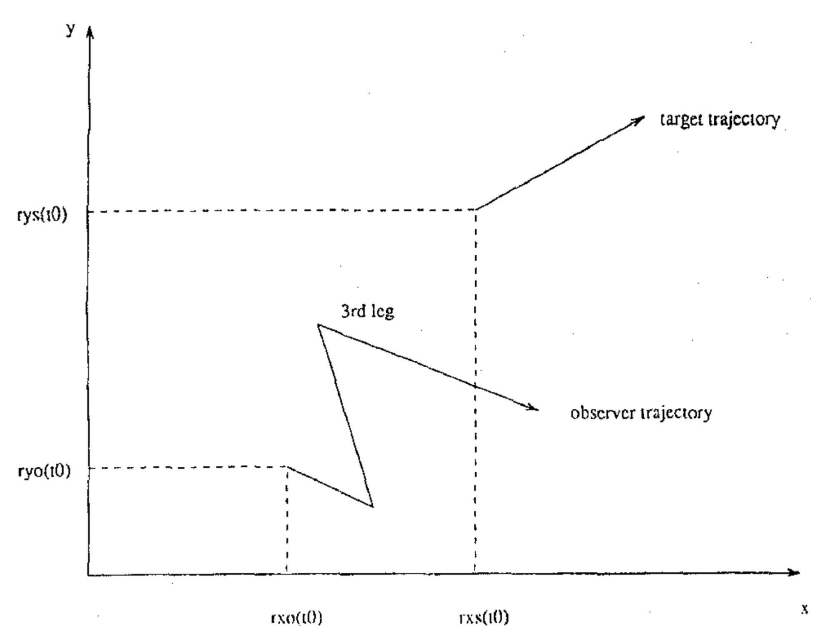

Fig. 1. Source-observer encounter.

properties of the $k$ estimates are considered in Section III.

Considering that the measurements include the spatial frequency rate estimates, Section IV deals with the estimation algorithms of source trajectory parameters, while the statistical properties of the extended TMA are considered in Section V. The calculation of the TMA lower bounds are then restricted to a special case study: nonmaneuvering observer. Beyond practical interest, simple formulations of the TMA lower bounds can be obtained for this special case. The calculation results are detailed in Section VI.

Simulation results are presented in Section VII, the improvements in TMA obtained by extending the measurements to spatial-frequency rate estimates $(\hat{\dot{k}})$ are carefully considered. Finally, Section VIII deals with multiple source TMA. For that purpose, an original method is presented.

\section{A SIMPLIFIED MODEL OF SOURCE MOTION SEEN BY THE ARRAY}

The notations are those of [1]. Consider the source-observer encounter depicted in Fig. 1. The source, located at the coordinates $\left(r_{x s}, r_{y s}\right)$ moves with constant velocities $\left(v_{x s}, v_{y s}\right)$ and is thus defined to have the state vector:

$$
X_{s} \stackrel{\Delta}{=}\left[r_{x s}, r_{y s}, v_{x s}, v_{y s}\right]^{\mathrm{T}}
$$

with $\mathrm{T}$ defining matrix transposition.

The observer state is similarly defined as

$$
X_{0} \triangleq\left[r_{x 0}, r_{y 0}, v_{x 0}, v_{y 0}\right]^{\mathrm{T}}
$$

in terms of the relative state vector $X$, defined by

$$
X=X_{s}-X_{0} \triangleq\left[r_{x}, r_{y}, v_{x}, v_{y}\right]^{\mathrm{T}} .
$$

The discrete time equation takes the following form:

$$
X\left(t_{k}\right)=\Phi\left(t_{k}, t_{k-1}\right) X\left(t_{k-1}\right)+U\left(t_{k}\right)
$$

where

$$
\begin{aligned}
\Phi\left(t_{k}, t_{k-1}\right) & =\left(\begin{array}{cc}
I d & \left(t_{k}-t_{k-1}\right) I d \\
O & I d
\end{array}\right), \\
I d & \triangleq\left(\begin{array}{ll}
1 & 0 \\
0 & 1
\end{array}\right)
\end{aligned}
$$

and $t_{k}$ is the time at the $k$ th sample, and the vector $U\left(t_{k}\right)=\left(0,0, u_{x}\left(t_{k}\right), u_{y}\left(t_{k}\right)\right)^{\mathrm{T}}$ accounts for the effects of observer accelerations.

Classically, the available measurements are the estimated angles $\theta_{t}$ (bearings) from the observer's platform to the source or, equivalently, the estimated values of the spatial frequencies $k_{t}\left(k_{t}=\sin \theta_{t} / \lambda, \lambda\right.$; wavelength), so that:

$$
\hat{k}_{t}=k_{t}+\nu_{t}
$$

where $\nu_{t}$ is a zero-mean independent Gaussian noise with variance $\sigma_{\nu}^{2}$, and $k_{t}$ is related to the state vector by the following equation:

$$
\begin{aligned}
k_{t} & =\left(\sin \theta_{t}\right) / \lambda \\
\theta_{t} & =\tan ^{-1}\left(\frac{r_{x}(t)}{r_{y}(t)}\right) .
\end{aligned}
$$

For a spatially isolated source, the variance $\sigma_{\nu}^{2}$ is given by the following formula, classical in the array processing literature and known as Woodward's formula $[3,8]$ :

$$
\sigma_{\nu}^{2}=\frac{3(1+p \rho)(2 N-1)}{\rho^{2} p^{2}\left(p^{2}-1\right) \pi^{2} d^{2} N(N+1)}
$$

with

$$
\mid \begin{aligned}
& \rho: \text { signal to noise ratio } \\
& p: \text { sensor number } \\
& N: \text { number of snapshots } \\
& d: \text { intersensor distance }
\end{aligned}
$$

(source in the array broadside).

We stress that the above formula is valid only for a linear array regularly sampled (in space). This is the array configuration for the sequel. This choice is due to the simplicity of spatio-temporal analysis for this array geometry as well as to practical considerations.

The various factors defining $\sigma_{\nu}^{2}$ (eq. (6)) need some comments, especially the factor $N$ which conditions the measurements statistics and thus the TMA performance. It is limited by the nonstationary nature of the TMA problem. An optimal value of $N\left(N_{\text {opt }}\right)$ can be determined [6], conditioned by the sensor number $p$ and the source trajectory. Thus an increase in $p$ reduces $N_{\text {opt }}$. Actually, the variance of $\hat{k}$ cannot be reduced below a certain value. In the spirit of spatio-temporal analysis, a new parameter (named $k$ ) is presented. 


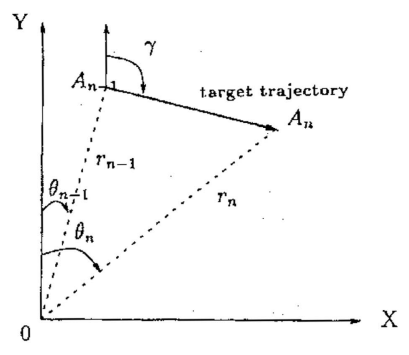

Fig. 2. Relative source motion.

Consider, now, the relative source motion depicted in Fig. 2, the source heading $\gamma$ is defined WRT the north. With the notations of Fig. 2, the following equations result from elementary trigonometry

$$
r_{j} \sin \theta_{j}=r_{j-1} \sin \left(\theta_{j-1}\right)+(\sin \gamma) v \Delta T
$$

(equality of the projections on the north axis),

$$
r_{j}^{2}=r_{j-1}^{2}+(v \Delta T)^{2}+2 r_{j-1} \cos \left(\theta_{j-1}-\gamma\right) v \Delta T
$$

(equality of the norms), with

$$
\begin{aligned}
\cos \gamma & =\frac{v_{y}}{v} \quad \text { and } \quad \sin \gamma=\frac{v_{x}}{v} \\
\Delta T & : \text { sampling time }
\end{aligned}
$$

The sampling time is the time corresponding to a snapshot. The relative velocity vector is assumed to be constant, this hypothesis is justified on a leg [6]. On a leg, the previous relations are valid for any value of the time index $(n)$, yielding

$$
\begin{aligned}
r_{n} \sin \theta_{n} & =r_{0} \sin \left(\theta_{n-1}\right)+n v \Delta T \sin \gamma \\
r_{n}^{2} & =r_{0}^{2}+(n v \Delta T)^{2}+2 n r_{0} v \Delta \cos \left(\theta_{0}-\gamma\right) .
\end{aligned}
$$

Denoting $k_{n}$ the instantaneous spatial frequency (i.e., $k_{n}=\sin \theta_{n} / \lambda$ : wavelength), the following equality can thus be deduced:

$$
k_{n}=\frac{r_{0} \sin \theta_{0}+n v \Delta T \sin \gamma}{\lambda\left[r_{0}^{2}+(n v \Delta T)^{2}+2 n r_{0} v \Delta T \cos \left(\gamma-\theta_{0}\right)\right]^{1 / 2}} .
$$

Considering the increment $x=n v \Delta T / r_{0}$, then the following first-order expansion of $k_{n}$ is directly deduced from (9):

$$
\begin{aligned}
& k_{n}=\frac{\sin \theta_{0}+x \sin \gamma}{\lambda\left(1+2 x \cos \left(\theta_{0}-\gamma\right)+x^{2}\right)^{1 / 2}} \\
& \quad \stackrel{1}{=} k_{0}+n \dot{k}
\end{aligned}
$$

with

$$
\begin{aligned}
\dot{k} & =\left[\sin \gamma-\sin \theta_{0} \cos \left(\theta_{0}-\gamma\right)\right] \frac{v \Delta T}{r_{0}} \\
& =\cos \theta_{0} \sin \left(\gamma-\theta_{0}\right) \frac{v \Delta T}{\lambda r_{0}} .
\end{aligned}
$$

The parameter $\dot{k}$ represents the (approximated) first-order derivative of the spatial frequency.
Obviously, the validity of this approximation is conditioned by the value of the increment $x$, itself depending upon the values of $n, v$, and $r_{0}$. Its validity domain increases with $r_{0}$. The parameter $k$ is a nonlinear function of the state vector $X_{0}$ (eq. (3)). This dependence is now explained. More precisely, one has from (7), (10):

$$
\begin{aligned}
\dot{k} & =\left[\frac{v_{x}}{v}-\left(\frac{v_{y}}{v} \frac{r_{y}}{r_{0}}+\frac{v_{x}}{v} \frac{r_{x}}{r_{0}}\right) \frac{r_{x}}{r_{0}}\right] \frac{v \Delta T}{\lambda r_{0}} \\
& =\frac{r_{y}}{\lambda r_{0}^{3}} \Delta T\left[r_{y} v_{x}-r_{x} v_{y}\right]
\end{aligned}
$$

where the time index $t$ is omitted.

Since this formula is essential for the sequel, let us detail it briefly. Obviously it may obtained directly from (10) by using the following substitutions:

$$
\begin{aligned}
\sin \gamma & =\frac{v_{x}}{v}, & \cos \gamma & =\frac{v_{y}}{v} \\
\sin \theta_{0} & =\frac{r_{x}}{r}, & \cos \theta_{0} & =\frac{r_{y}}{r} .
\end{aligned}
$$

A perhaps more direct approach is

$$
\theta=\tan ^{-1}\left(\frac{r_{x}}{r_{y}}\right)
$$

and therefore

$$
\dot{\theta}=\frac{v_{x} r_{y}-r_{x} v_{y}}{r_{y}^{2}} \cos ^{2} \theta=\frac{v_{x} r_{y}-r_{x} v_{y}}{r^{2}}
$$

so that finally,

$$
\dot{k}=\frac{\cos \theta}{\lambda} \dot{\theta} \Delta T=\frac{r_{y}}{\lambda r^{3}}\left(v_{x} r_{y}-r_{x} v_{y}\right) \Delta T .
$$

Further, note the following expression of $\dot{k}$ :

$$
\dot{k}=\frac{r_{y}}{\lambda r_{0}^{3}} \operatorname{det}\left(\begin{array}{ll}
v_{x} & r_{x} \\
v_{y} & r_{y}
\end{array}\right)
$$

(det meaning determinant) which provides a geometric interpretation of the parameter $\dot{k}$.

The above expression (11) is instrumental for the inclusion of the parameter $k$ in the TMA algorithms. Using the notations of [1], denote $t_{m}$ the reference time for the state vector, then one has directly:

$$
\mid \begin{aligned}
& r_{x}\left(t_{j}\right)=r_{x}\left(t_{m}\right)+\left(t_{j}-t_{m}\right) v_{x}\left(t_{m}\right) \\
& r_{y}\left(t_{j}\right)=r_{y}\left(t_{m}\right)+\left(t_{j}-t_{m}\right) v_{y}\left(t_{m}\right)
\end{aligned}
$$

with

$$
\mid \begin{aligned}
& v_{x}\left(t_{m}\right)=v_{x s}\left(t_{m}\right)-v_{x 0}\left(t_{m}\right) \\
& v_{y}\left(t_{m}\right)=v_{y s}\left(t_{m}\right)-v_{y 0}\left(t_{m}\right)
\end{aligned}
$$

where $v_{x s}, v_{y s}$ are assumed to be constant and $v_{x 0}$ and $v_{y 0}$ are known. Furthermore, the following relations hold (the source velocity vector is constant):

$$
\begin{aligned}
v_{x}\left(t_{j}\right) & =v_{x}\left(t_{m}\right)+\left(v_{x 0}\left(t_{m}\right)-v_{x 0}\left(t_{j}\right)\right) \\
& =v_{x}\left(t_{m}\right)+\alpha_{x}\left(t_{m}\right) \\
v_{y}\left(t_{j}\right) & =v_{y}\left(t_{m}\right)+\alpha_{y}\left(t_{m}\right) .
\end{aligned}
$$


The terms $\alpha_{x}$ and $\alpha_{y}$ in (13) depend only upon the observer maneuver. The state vector is now the vector $X_{m}$ defined by

$$
X_{m}^{\mathrm{T}}=\left[r_{x}\left(t_{m}\right), r_{y}\left(t_{m}\right), v_{x}\left(t_{m}\right), v_{y}\left(t_{m}\right)\right]^{\mathrm{T}} .
$$

Using (11)-(13) the parameter $\dot{k}_{j}$ (denoting the value of $\dot{k}$ for $t=t_{j}$ ) can be expressed in terms of the state components:

$$
\dot{k}_{j}=\frac{r_{y}\left(t_{j}\right)}{\lambda r_{j}^{3}} \Delta T\left[r_{y}\left(t_{j}\right) v_{x}\left(t_{j}\right)-r_{x}\left(t_{j}\right) v_{y}\left(t_{j}\right)\right]
$$

with

$$
r_{j} \triangleq\left(r_{x}^{2}\left(t_{j}\right)+r_{y}^{2}\left(t_{j}\right)\right)^{1 / 2} .
$$

The calculation of the partial derivatives of $\dot{k}_{j}$ WRT the state components is direct, yielding

$$
\begin{aligned}
\frac{\partial \dot{k}_{j}}{\partial r_{x}\left(t_{m}\right)}= & -\frac{\cos \theta_{j} \Delta T}{\lambda r_{j}^{2}}\left[v_{y}\left(t_{j}\right)\left(1-3 \sin ^{2} \theta_{j}\right)+v_{x}\left(t_{j}\right)\left(3 \sin \theta_{j} \cos \theta_{j}\right)\right] \\
\frac{\partial \dot{k}_{j}}{\partial r_{y}\left(t_{m}\right)}= & -\frac{\Delta t}{\lambda r_{j}^{2}}\left[v_{x}\left(t_{j}\right) \cos \theta_{j}\left(1-3 \sin ^{2} \theta_{j}\right)\right. \\
& \left.\quad+v_{y}\left(t_{j}\right) \sin \theta_{j}\left(1-3 \cos ^{2} \theta_{j}\right)\right] \\
\frac{\partial \dot{k}_{j}}{\partial v_{x}\left(t_{m}\right)}= & \frac{\cos ^{2} \theta_{j} \Delta T}{\lambda r_{j}}-\left(t_{j}-t_{m}\right) \frac{\cos \theta_{j} \Delta T}{\lambda r_{j}^{2}} \\
& \times\left[v_{y}\left(t_{j}\right)\left(1-3 \sin ^{2} \theta_{j}\right)+v_{x}\left(3 \sin \theta_{j} \cos \theta_{j}\right)\right] \\
\frac{\partial \dot{k}_{j}}{\partial v_{y}\left(t_{m}\right)}= & -\frac{\sin \theta_{j} \cos \theta_{j} \Delta T}{\lambda r_{j}}-\left(t_{j}-t_{m}\right) \frac{\Delta T}{\lambda r_{j}^{2}} \\
& \times\left[v_{x}\left(t_{j}\right) \cos \theta_{j}\left(1-3 \sin ^{2} \theta_{j}\right)+v_{y}\left(t_{j}\right) \sin \theta_{j}\left(1-3 \cos ^{2} \theta_{j}\right)\right]
\end{aligned}
$$

with

$$
\cos \theta_{j} \triangleq \frac{r_{y}\left(t_{j}\right)}{r_{j}} \quad \text { and } \quad \sin \theta_{j} \triangleq \frac{r_{x}\left(t_{j}\right)}{r_{j}} .
$$

The above formulas are instrumental for the inclusion of the parameter $\dot{k}$ in the TMA algorithm. Let us now focus our attention on the estimation of this parameter.

\section{ON ESTIMATION OF PARAMETER $k$}

The lower bounds for the variance of estimation of the parameters $k_{0}$ and $\dot{k}$ are investigated. Considering the unique source case and the observations made up of the array snapshots $V_{t}$, the calculations take a classical form [7], i.e.,

$$
\begin{aligned}
\mathcal{L} & \triangleq \log \left[p\left(V_{1}, V_{2}, \ldots, V_{N}\right) /\left(k_{0}, \dot{k}\right)\right] \\
& =-N p \log \pi-\sum_{t=1}^{N} \log \operatorname{det} \Gamma_{t}-\sum_{t=1}^{N} \operatorname{tr}\left(\Gamma_{t}^{-1} V_{t} V_{t}^{*}\right)
\end{aligned}
$$

with $\Gamma_{t} \triangleq \operatorname{cov}\left(V_{t}\right)$, the symbols tr and det denote, respectively, the trace and the determinant, $p$ is the sensor number.

The functional $\mathcal{L}$ is the likelihood functional associated with the observation vectors $V_{t}$ which are assumed to be circular gaussian [9] and uncorrelated $\left(t \neq t^{\prime}\right)$. The density of the circular Gaussian vector $V_{t}$ is given by

$$
V_{t}: \mathcal{N}_{\mathbb{C}}\left(0, \Gamma_{t}\right)
$$

with

$$
\Gamma_{t}=\rho D_{k_{t}} D_{k_{t}}^{*}+I d
$$

where $I d$ is the identity matrix, $\rho$ is the signal/noise ratio, and $D$ is the steering vector [3].

The Fisher information matrix (FIM) relative to the estimation of the two parameters $k_{0}$ and $\dot{k}$ is defined by

$$
\begin{gathered}
F=\left(F_{i j}\right), \\
F_{11}=-\mathbb{E}\left(\frac{\partial^{2} \mathcal{L}}{\partial k_{0}^{2}}\right), \\
F_{12}=-\mathbb{E}\left(\frac{\partial^{2} \mathcal{L}}{\partial k_{0} \partial \dot{k}}\right), \\
F_{22}=-\mathbb{E}\left(\frac{\partial^{2} \mathcal{L}}{\partial \dot{k}^{2}}\right) .
\end{gathered}
$$

The elements of the FIM are calculated by means of the following classical formulas (Bang's formula [3]):

$$
\begin{aligned}
& F_{11}=\sum_{t=1}^{N} \operatorname{tr}\left[\Gamma_{t}^{-1} \frac{\partial \Gamma_{t}}{\partial k_{0}} \Gamma_{t}^{-1} \frac{\partial \Gamma_{t}}{\partial k_{0}}\right], \\
& F_{12}=\sum_{t=1}^{N} \operatorname{tr}\left[\Gamma_{t}^{-1} \frac{\partial \Gamma_{t}}{\partial k_{0}} \Gamma_{t}^{-1} \frac{\partial \Gamma_{t}}{\partial \dot{k}}\right] .
\end{aligned}
$$

At this point, it is worth noting the following relations:

$$
\begin{aligned}
\operatorname{tr}\left[\Gamma_{t}^{-1} \frac{\partial \Gamma_{t}}{\partial \dot{k}} \Gamma_{t}^{-1} \frac{\partial \Gamma_{t}}{\partial \dot{k}}\right] & =t \operatorname{tr}\left[\Gamma_{t}^{-1} \frac{\partial \Gamma_{t}}{\partial \dot{k}} \Gamma_{t}^{-1} \frac{\partial \Gamma_{t}}{\partial k_{0}}\right] \\
& =t^{2} \operatorname{tr}\left[\Gamma_{t}^{-1} \frac{\partial \Gamma_{t}}{\partial k_{0}} \Gamma_{t}^{-1} \frac{\partial \Gamma_{t}}{\partial k_{0}}\right] .
\end{aligned}
$$

Consequently, the FIM calculation is reduced to the calculation of $F_{11}$ which is straightforwardly obtained, i.e.,

$$
F_{11}=\frac{N \rho^{2} 4 \pi^{2} d^{2} p^{2}\left(p^{2}-1\right)}{6(1+p \rho)}=N \Theta_{11}
$$

and, then

$$
\begin{aligned}
& F_{22}=\frac{N(N+1)(2 N+1)}{6} \Theta_{11}, \\
& F_{12}=\frac{N(N+1)}{2} \Theta_{11} .
\end{aligned}
$$


The lower bounds for $\operatorname{var}\left(\hat{k}_{0}\right)$ and $\operatorname{var}(\hat{\dot{k}})$ are directly deduced from (19), yielding

$$
\begin{aligned}
& \operatorname{var}\left(\hat{k}_{0}\right) \geq \frac{3(1+p \rho)(2 N-1)}{\rho^{2} p^{2}\left(p^{2}-1\right) \pi^{2} d^{2} N(N+1)} \\
& \operatorname{var}(\hat{\dot{k}}) \geq \frac{18(1+p \rho)}{\rho^{2} p^{2}\left(p^{2}-1\right) \pi^{2} d^{2}\left(N^{3}-N\right)} .
\end{aligned}
$$

The above formula is interesting since it proves that a lower bound of $\operatorname{var}\left(\hat{k}_{0}\right)$ is proportional to $N^{-1}$ while $\operatorname{var}(\hat{\dot{k}})$ depends on $N$ with a factor of $N^{-3}$. However, this result must be seriously mitigated since the value of $k$ is usually very small (e.g., $10^{-5}$ ). This result agrees with the naive calculation of $\operatorname{var}(\hat{\dot{k}})$. More precisely, consider a moving source whose spatial frequencies are modeled by a linear model (i.e., $\left.k_{t}=k_{0}+t \dot{k}\right)$, using the sequence of snapshot vectors $\left\{V_{t}\right\}_{1}^{N}$ a sequence $\left\{\hat{k}_{t}\right\}$ of instantaneous (1 snapshot) spatial frequencies is estimated.

Then from (20), one obtains:

$$
\operatorname{var}\left(\hat{k}_{t}\right)=\frac{3(1+p \rho)}{2(\pi d \rho)^{2} p^{2}\left(p^{2}-1\right)} \triangleq \sigma_{k}^{2} .
$$

Consider now the vector $\mathcal{X}$ of estimated spatial frequencies, i.e.,

$$
\mathcal{X}^{T}=\left(\hat{k}_{1}, \ldots, \hat{k}_{N}\right)
$$

then, assuming its Gaussianity:

with

$$
\mathcal{X} \text { is } \mathcal{N}\left(M, \sigma_{k}^{2} I d\right)
$$

$$
M^{\mathrm{T}}=\left(k_{0}, k_{0}+\dot{k}, \ldots, k_{0}+(N-1) \dot{k}\right) .
$$

Then, using (22) and (23), the variance of $\hat{\dot{k}}$ is obtained straightforwardly and coincides with (21). It is stressed that the natures of the two calculations of the bounds are essentially different since the first one deals directly with the spatio-temporal data while the second corresponds to a postprocessing. Thus, in the second case, the estimation of $\dot{k}$ does not contain any extraneous information WRT the sequence of $\left\{k_{t}\right\}$. Fortunately, this is not true for the first approach.

It is possible to consider simultaneously the estimation of the two parameters $k_{0}$ and $\dot{k}$, the more natural approach seems then the 2D (in $k_{0}$ and $\dot{k}$ ) focused beamforming method $[3,7]$. However, this approach suffers from serious drawbacks like interference between sources, spurious peaks, etc. The modeling of the spatio-temporal data by a multiscale state space model [7] seems to be a more promising way. More precisely, let $\hat{R}_{t}$ the estimated CSM (cross spectral matrix) be defined as follows:

$$
\hat{R}_{t}=V_{t} V_{t}^{*}
$$

then $\hat{R}_{t}$ is projected on the Toeplitz subspace [10] by means of the classical algorithm (the projected matrix is denoted $\hat{R}_{t, T}$ ), yielding:

$$
1^{-s t} \operatorname{row} \hat{R}_{t, T} \triangleq\left(\hat{r}_{0}(t), \hat{r}_{1}(t), \ldots, \hat{r}_{p-1}(t)\right)
$$

with

$$
\hat{r}_{i}(t)=\frac{1}{p-i} \operatorname{tr}\left(\hat{R}_{t} Z^{i}\right) \quad 0 \leq i \leq p-1
$$

$Z^{i}$ being the $i$ th power of the shift matrix $Z$, i.e.,

$$
Z(m, n)= \begin{cases}1 & \text { if } m-n=1 \\ 0 & \text { otherwise. }\end{cases}
$$

This projection is quite classical in the array processing area, it amounts to a structured estimation of the covariance matrix (Toeplitz structure) $[7,11]$ and, generally, improves the angular resolution [12, 13]. Then, the spatio-temporal data are constituted of the two-dimensional (2D) spatio-temporal data $y(t, m)$ defined below:

$$
y(t, m)=\hat{r}_{m}(t) .
$$

Then, using (10) and (18) the 2D array of data $y(t, m)$ can be represented by the following $2 \mathrm{D}$ state space model [7]:

$$
\begin{cases}X(t+1, m)=F_{1}^{m} X(t, m) & 1 \leq m \leq p-1 \\ X(t, m+1)=F_{0} F_{1}^{t} X(t, m) & \\ y(t, m)=h^{*} X(t, m)+w(t, m) & t_{0} \leq t \leq t_{e}\end{cases}
$$

where $F_{1}^{m}$ and $F_{1}^{t}$ are, respectively, the $m$ th and $t$ th power of $F_{1}$ with

$$
\mid \begin{aligned}
& F_{1}=\operatorname{diag}\left(\exp \left(2 i \pi d \dot{k}_{1}\right), \ldots, \exp \left(2 i \pi d \dot{k}_{s}\right)\right) \\
& F_{0}=\operatorname{diag}\left(\exp \left(2 i \pi d k_{1,0}\right), \ldots, \exp \left(2 i \pi d k_{s, 0}\right)\right) \\
& h^{*}=(1,1, \ldots, 1) \\
& s: \text { source number. }
\end{aligned}
$$

Actually this 2D modeling simply results from the expression of the exact spatio-temporal covariances $r_{m}(t)$ (eq. (25)) i.e.,

$$
r_{m}(t)=\sum_{j=1}^{s} \sigma_{j}^{2} \exp \left[2 i \pi d m\left(k_{j, 0}+t \dot{k}_{j}\right)\right] .
$$

Using (24) and elementary linear algebra, the noise statistic is easily obtained:

$$
\begin{aligned}
\mathbb{E}(w(t, m)) & =0, \\
\mathbb{E}\left(w\left(t, m_{1}\right) w^{*}\left(t, m_{2}\right)\right) & =\frac{\operatorname{tr}\left(R_{t}^{\downarrow m_{1}} R_{t}^{\uparrow m_{2}}\right)}{\left(p-m_{1}\right)\left(p-m_{2}\right)}
\end{aligned}
$$

with

$$
\mid \begin{aligned}
& \text { tr denoting the trace of a matrix } \\
& R_{t}^{\left\lfloor m_{1}\right.} \triangleq Z^{m_{1}} R_{t}, R_{t}^{\dagger m_{2}} \triangleq\left(Z^{m_{2}}\right)^{\mathrm{T}} R_{t} .
\end{aligned}
$$

Despite its simplicity, this model is quite enlightening since it demonstrates the interest of the notion of spatio-temporal diversity. In particular, it is evident from (26) that the transition matrix $F_{1}$ will 
play a fundamental role. Furthermore, the transition matrices $F_{0}$ and $F_{1}$ may be separately estimated. It is then possible to replace the notion of instantaneous bearings by the source trajectory one, allowing thus to consider long integration time. For that purpose, original approaches have been developed [7, 28]. As they appear fundamental for multiple source TMA, they are presented in Section VIII. But let us now focus our attention on the estimation of the source state vector.

\section{ESTIMATION ALGORITHMS OF SOURCE STATE VECTOR}

The methods presented above, provide separate estimations of the parameters $k$ and $\dot{k}$. The

estimated values $\hat{\dot{k}}$ may thus be added to the classical measurements $\hat{k}_{t}$.

Given the history of measured spatial frequencies $\hat{k}_{j}$ (i.e., $\left.\hat{\mathbf{K}} \triangleq\left(\hat{k}_{1}, \ldots, \hat{k}_{N}\right)^{\mathrm{T}}\right)$ and the estimated values $\hat{\dot{k}}_{j}$ (i.e., $\hat{\dot{K}} \triangleq\left(\hat{\dot{k}}, \ldots, \hat{\dot{k}}_{L}\right)$, e.g., $L=$ number of legs), the likelihood functional conditional on the state vector $X$ stands as follows:

$$
p(\mathcal{K} \mid X)=\text { const. } \exp \left[-\frac{1}{2}(\hat{\mathcal{K}}-\mathcal{K})^{\mathrm{T}} W^{-1}(\hat{\mathcal{K}}-\mathcal{K})\right]
$$

with

$$
\hat{\mathcal{K}} \triangleq\left(\frac{\hat{\mathbf{K}}}{\hat{\hat{\mathbf{K}}}}\right), \quad \hat{\mathcal{K}}(X) \triangleq\left(\frac{\mathbf{K}(\mathbf{X})}{\dot{\mathbf{K}}(\mathbf{X})}\right)
$$

the covariance matrix $W$ is deduced from (19)-(21) and

$$
X_{m}=\left(r_{x}\left(t_{m}\right), r_{y}\left(t_{m}\right), v_{x}\left(t_{m}\right), v_{y}\left(t_{m}\right)\right)^{\mathrm{T}} \quad(\text { see }(14))
$$

Using (1)-(5), the matrix of partial derivatives of the extended measurement vector takes the following form [1]:

$$
\frac{\partial \mathbf{K}(X)}{\partial X}=\Delta T\left(\begin{array}{ccc}
\frac{\cos ^{2} \theta_{1}}{\lambda r_{1}}, & \frac{-\sin \theta_{1} \cos \theta_{1}}{\lambda r_{1}}, & \left(t_{1}-t_{m}\right) \frac{\cos ^{2} \theta_{1}}{\lambda r_{1}},-\left(t_{1}-t_{m}\right) \frac{\sin \theta_{1} \cos \theta_{1}}{\lambda r_{1}} \\
\vdots & \vdots & \vdots \\
\frac{\cos ^{2} \theta_{N}}{\lambda r_{N}}, & \frac{-\sin \theta_{N} \cos \theta_{N}}{\lambda r_{N}}, & \left(t_{N}-t_{m}\right) \frac{\cos ^{2} \theta_{N}}{\lambda r_{N}},-\left(t_{N}-t_{m}\right) \frac{\sin \theta_{N} \cos \theta_{N}}{\lambda r_{N}}
\end{array}\right)
$$

while the elements of the matrix $\partial \dot{\mathbf{K}} / \partial X$ are given by (16).

A Gauss-Newton algorithm [1,14] for the maximization of the likelihood functional is easily deduced from (28) and takes the following form:

$$
\begin{aligned}
X_{\ell+1}= & X_{\ell}-\rho_{\ell}\left[\left(\frac{\partial \mathcal{K}}{\partial \mathbf{X}}\right)^{\mathrm{T}} W^{-1}\left(\frac{\partial \mathcal{K}}{\partial \mathbf{X}}\right)\right]^{-1} \\
& \times\left(\frac{\partial \mathcal{K}}{\partial \mathbf{X}}\right)^{\mathrm{T}} W^{-1}(\hat{\mathcal{K}}-\mathcal{K})
\end{aligned}
$$

where $\mathcal{K}$ and $\partial \mathcal{K} / \partial \mathbf{X}$ are evaluated at $X_{\ell}$ and the step size $\rho_{\ell}$ is selected at each iteration to ensure convergence.

Note that the observations $\hat{k}$ and $\hat{k}$ being assumed independent (they are not estimated on the same data), the noise matrix $W$ is (approximately) diagonal. This assumption is considered for the rest and especially for all the simulation results. Even if the introduction of a nondiagonal matrix $W$ does not induce any theoretical difficult, it is not a more realistic hypothesis.

\section{STATISTICAL PROPERTIES OF EXTENDED TMA}

Similar to [1], a uniform sampling rate in time for $k\left(t_{i}\right)$ and $k\left(t_{j}\right)$ (note that they are generally quite different) is assumed that allows the substitution of $i$ for $t_{i}$ or $t_{j}$. The FIM takes the following form:

$$
\mathrm{FIM} \simeq\left(\frac{\partial \mathcal{K}}{\partial X}\right)^{\mathrm{T}} W^{-1}\left(\frac{\partial \mathcal{K}}{\partial X}\right)
$$

where the matrix $W$ can be roughly approximated (for a far source) by a diagonal matrix, i.e.,

$$
W \simeq\left(\begin{array}{cc}
\sigma_{k}^{2} I d_{N} & O \\
O & \dot{\sigma}_{k}^{2} I d_{L}
\end{array}\right) .
$$

The details of this matrix are revealed by partitioning it into $2 \times 2$ submatrices to obtain the form [1] $(\Delta T=1)$ :

$$
\mathrm{FIM}=\operatorname{FIM}(\mathbf{K})+\operatorname{FIM}(\dot{\mathbf{K}})
$$

with

$$
\operatorname{FIM}(\mathbf{K}) \simeq \frac{1}{\left(\sigma_{k} r \lambda\right)^{2}} \sum_{i=1}^{n}\left(\begin{array}{cc}
\Omega_{i} & (i-m) \Omega_{i} \\
(i-m) \Omega_{i} & (i-m)^{2} \Omega_{i}
\end{array}\right)
$$


and

$$
\Omega_{i}=\cos ^{2} \theta_{i}\left(\begin{array}{cc}
\cos ^{2} \theta_{i} & -\frac{1}{2} \sin 2 \theta_{i} \\
-\frac{1}{2} \sin 2 \theta_{i} & \sin ^{2} \theta_{i}
\end{array}\right) .
$$

The first and second components of the vector $\partial \dot{k} / \partial X$ (eq. (16)) (i.e., $\partial \dot{k} / \partial r_{x}$ and $\partial \dot{k} / \partial r_{y}$ ) are little WRT the two last components, therefore

$$
\frac{\partial \dot{k}_{j}}{\partial X} \simeq\left(0,0, \frac{\partial \dot{k}}{\partial v_{x, m}}, \frac{\partial \dot{k}}{\partial v_{y, m}}\right)^{\mathrm{T}} .
$$

A further step of approximation consists in approximating $\partial \dot{k} / \partial v_{x}$ and $\partial \dot{k} / \partial v_{y}$, i.e.,

$$
\frac{\partial \dot{k}}{\partial v_{x}} \simeq \frac{\cos ^{2} \theta_{j}}{\lambda r_{j}}, \quad \frac{\partial \dot{k}}{\partial v_{y}} \simeq-\frac{1}{2} \frac{\sin 2 \theta_{j}}{\lambda r_{j}}
$$

so that

$$
\operatorname{FIM}\left(\dot{k}_{t}\right) \simeq \frac{1}{\left(\sigma_{k} \lambda r\right)^{2}} \sum_{j=1}^{L}\left(\begin{array}{cc}
O & O \\
O & \Omega_{j}^{\prime}
\end{array}\right)
$$

with

$$
\Omega_{j}^{\prime}=\cos ^{2} \theta_{j}\left(\begin{array}{cc}
\cos ^{2} \theta_{j} & -\frac{1}{2} \sin \left(2 \theta_{j}\right) \\
-\frac{1}{2} \sin \left(2 \theta_{j}\right) & \sin ^{2} \theta_{j}
\end{array}\right)
$$

where $r_{i}$ is approximated by the constant $r$.

This last expression reveals that, at a first glance, it is mainly the estimation of the source velocity which may be improved by using the extended vector of measurements $\mathcal{K}$. Actually, the effect of $k_{t}$ increases with the values taken by the factor $(i-m)$ in $\operatorname{FIM}(\mathbf{K})$. However, on another hand, this effect is mitigated by the respective values of $\sigma_{k}^{2}$ and $\sigma_{\dot{k}}^{2}$ (eq. (21)). The inclusion of the vector $\mathbf{K}$ in the measurement vector can thus seriously improve the estimation of the source velocity. This point is detailed later for a special case study (see Section VI).

\section{A SPECIAL CASE STUDY}

The utilization of a large linear towed array can seriously restrict the ownship maneuvers. The ownship motion is then rectilinear and with a constant velocity. It is well known that the bearing-only TMA problem is unobservable in this case $[15,16]$, but the observability may be recovered by adding extraneous measurements like Doppler shifts [17-19].

However, the temporal variations of the source frequency are, generally, very weak for far sources, which reduces the practical interest of these extraneous measurements.

Using the bearings-only measurements, the TMA problem is partially observable since the observability gramian is rank deficient in the absence of ownship maneuver [15]. This is true even if the spatial frequency rate $\hat{\dot{k}}$ is added. A way for overcoming this problem consists of a parametrization of the solution space. More precisely, the (partial) state $X_{m, p}$ is defined as follows (eq. (14)):

$$
X_{m, p}=\left[r_{y}\left(t_{m}\right), v_{x}\left(t_{m}\right), v_{y}\left(t_{m}\right)\right]^{\mathrm{T}} .
$$

Note that this choice of the partial state $X_{m, p}$ is arbitrary; it may be any other three-dimensional vector whose components are convenient function of the whole state vector $X_{m}$ (e.g., $X_{m, p}^{\prime}=$ $\left.\left[r_{x}\left(t_{m}\right), r_{y}\left(t_{m}\right), v\left(t_{m}\right)\right]^{\mathrm{T}}\right)$.

For a given distance $r\left(t_{m}\right)$ between the source and the observer, the relative source-observer positions are given (at any time $t$ ) by

$$
\left\{\begin{array}{l}
r_{x}(t)=\left(r^{2}\left(t_{m}\right)-r_{y}^{2}\left(t_{m}\right)\right)^{1 / 2}+\left(t-t_{m}\right) v_{x}\left(t_{m}\right) \\
r_{y}(t)=r_{y}\left(t_{m}\right)+\left(t-t_{m}\right) v_{y}\left(t_{m}\right)
\end{array}\right.
$$

so that the elements of the matrix of the partial derivatives of the extended measurement vector $\mathcal{K}$ (eqs. (16), (28)) stand as follows.

1)

$$
\frac{\partial k_{i}}{\partial r_{y}\left(t_{m}\right)}=-\frac{\Delta T \cos \theta_{i}}{r_{i}}\left[\frac{\cos \theta_{i}}{\tan \theta_{m}}+\sin \theta_{i}\right] .
$$

2)

$$
\begin{aligned}
\frac{\partial \dot{k}_{i}}{\partial r_{y}\left(t_{m}\right)} & =\frac{\Delta T}{\lambda r_{i}^{2}}\left[v_{x} \cos \theta_{i}\left(\frac{3 \sin \theta_{i} \cos \theta_{i}}{\tan \theta_{m}}+3 \sin ^{2} \theta_{i}-1\right)\right. \\
& \left.-v_{y}\left\{\sin \theta_{i}\left(\frac{3 \sin \theta_{i} \cos \theta_{i}}{\tan \theta_{m}}+1-3 \cos ^{2} \theta_{i}\right)-\frac{\cos \theta_{i}}{\tan \theta_{m}}\right\}\right]
\end{aligned}
$$

3) The other elements (i.e., $\partial k_{i} / \partial v_{x}, \partial k_{i} / \partial v_{y}$, $\left.\partial \dot{k}_{i} / \partial v_{x}, \partial \dot{k}_{i} / \partial v_{y}\right)$ are identical to those of (16), (28).

The partial state $X_{m, p}(34)$ is then estimated by means of a Gauss-Newton algorithm similar to the previous one (eq. (29)). The initialization step requires a special procedure since the pseudolinear estimator (PLE) [1] is no longer appropriate since it is obtained by a linearization of the measurement equation. Here, the measurement $\dot{k}$ is a highly nonlinear function (eq. (15)) of the state and therefore the PLE (or MIV) estimator cannot be efficiently extended to this new measurement.

A simpler initialization procedure replaces the PLE. It is described below.

1) The distance $r\left(t_{m}\right)$ being given, approximate $\hat{\theta}\left(t_{m}\right)$ by a linear regression of the estimated bearings, then:

$$
\begin{aligned}
& \hat{r}_{x}\left(t_{m}\right)=r\left(t_{m}\right) \sin \left(\hat{\theta}\left(t_{m}\right)\right) \\
& \hat{r}_{y}\left(t_{m}\right)=r\left(t_{m}\right) \cos \left(\hat{\theta}\left(t_{m}\right)\right) .
\end{aligned}
$$



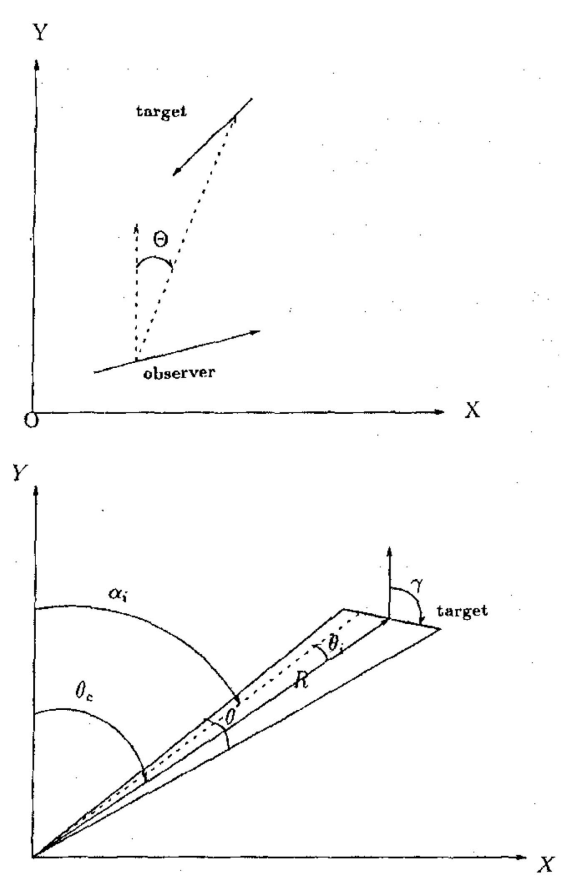

Fig. 3. TMA, partially observable case.

2)

$$
\hat{\mathbf{V}}=\left(A^{\mathrm{T}} A\right)^{-1} A^{\mathrm{T}} B \hat{\mathbf{R}}_{x y}\left(t_{m}\right)
$$

with

$$
\begin{aligned}
\hat{\mathbf{R}}_{x y}\left(t_{m}\right) & \triangleq\left|\begin{array}{cc}
\hat{r}_{x}\left(t_{m}\right) \\
\hat{r}_{y}\left(t_{m}\right)
\end{array}, \quad \hat{\mathbf{V}} \triangleq\right| \begin{array}{l}
\hat{v}_{x} \\
\hat{v}_{y}
\end{array} \\
A & =\left[\begin{array}{cc}
\vdots & \vdots \\
\left(t_{i}-t_{m}\right) \cos \hat{\beta}_{i} & -\left(t_{i}-t_{m}\right) \sin \hat{\beta}_{i} \\
\vdots & \vdots
\end{array}\right] \\
B & =\left[\begin{array}{cc}
\vdots & \vdots \\
\cos \hat{\beta}_{i} & -\sin \hat{\beta}_{i} \\
\vdots & \vdots
\end{array}\right]
\end{aligned}
$$

where $\left\{\hat{\beta}_{i}\right\}$ obtained by linear regression.

The above least-square estimate $\hat{V}$ comes from an approximated expansion of the bearing $\theta(t)(\theta(t)=$ $\left.\tan ^{-1}\left(r_{x}(t) / r_{y}(t)\right)\right)$.

This very rough approximation of the state vector provides a convenient initialization of the Gauss-Newton algorithms. The behavior of this algorithm is illustrated by simulation results (Section VII) but let us now focus our attention of the statistical analysis of this partially observable TMA problem.

For the sake of simplicity, the observer (ownship) is fixed and situated at the origin. This is not restrictive since in this partial TMA problem the observer velocity is constant. The observer-source geometry is depicted in Fig. 3 with the following geometric parameters [1]:

$$
\begin{aligned}
\theta & =\frac{N v \Delta T \sin \left(\gamma-\theta_{c}\right)}{r}=\frac{\text { baseline }}{\text { range }} \\
\alpha_{i} & =\theta_{c}+\frac{\theta}{2 N}(2 i-N) \\
& =\theta_{c}+\theta_{i}, \quad i=0, \ldots, N \\
\left(t_{i}-t_{m}\right) & =i \frac{\Delta T}{N}, \quad i=0, \ldots, N .
\end{aligned}
$$

The bearings-only TMA is considered in a first time. Then, the Fisher matrix (relative to $X_{m, p}$ ) takes the following form (from Section V):

with

$$
F=\frac{1}{\sigma_{\theta}^{2} r^{2}}\left(\begin{array}{ccc}
f_{1} & f_{2} & f_{3} \\
f_{2} & f_{4} & f_{5} \\
f_{3} & f_{5} & f_{6}
\end{array}\right)
$$

$$
\mid \begin{aligned}
& f_{1}=\sum_{i=0}^{N}\left(\frac{\cos \alpha_{i}}{\tan \theta_{m}}+\sin \alpha_{i}\right)^{2} \\
& f_{2}=\sum_{i}\left(t_{i}-t_{m}\right)\left(\frac{\cos ^{2} \alpha_{i}}{\tan \theta_{m}}+\sin \alpha_{i} \cos \alpha_{i}\right) \\
& f_{3}=\sum_{i}\left(t_{i}-t_{m}\right)\left(\frac{\cos \alpha_{i} \sin \alpha_{i}}{\tan \theta_{m}}+\sin ^{2} \alpha_{i}\right) \\
& f_{4}=\sum_{i}\left(t_{i}-t_{m}\right)^{2} \cos ^{2} \alpha_{i} \\
& f_{5}=-\sum_{i}\left(t_{i}-t_{m}\right)^{2} \cos _{i} \sin \alpha_{i} \\
& f_{6}=\sum_{i}\left(t_{i}-t_{m}\right)^{2} \sin ^{2} \alpha_{i} .
\end{aligned}
$$

An expansion (WRT $\theta_{i}$ ) of the basic expressions involved in the $\left\{f_{i}\right\}$ constitutes the basis of the statistical analysis. For instance, the second-order expansion of these basic expressions stands as follows:

$$
\begin{aligned}
\cos ^{2} \alpha_{i}= & \cos ^{2} \theta_{c}-\sin \left(2 \theta_{c}\right) \theta_{i}+\left(\sin ^{2} \theta_{c}-\cos ^{2} \theta_{c}\right) \theta_{i}^{2} \\
\sin ^{2} \alpha_{i}= & \sin ^{2} \theta_{c}+\sin \left(2 \theta_{c}\right) \theta_{i}+\left(\cos ^{2} \theta_{c}-\sin ^{2} \theta_{c}\right) \theta_{i}^{2} \\
\sin \alpha_{i} \cos \alpha_{i}= & \sin \theta_{c} \cos \theta_{c}+\left(\cos ^{2} \theta_{c}-\sin ^{2} \theta_{c}\right) \theta_{i} \\
& -\sin \left(2 \theta_{c}\right) \theta_{i}^{2} .
\end{aligned}
$$

The analysis of the following scenario has been considered in a first time. The ownship is fixed and situated at the origin. The source trajectory is rectilinear and symmetric WRT the $y$ axis; the distance $r$ is assumed to be constant. Note that, with the previous notations, this scenario corresponds to $\theta_{c}=0$. A high (5) order expansion of the FIM elements $\left\{f_{i}\right\}$ have been considered. Since the calculations are (rather) tedious, they have been achieved by means of the software MAPLE (Waterloo MAPLE Software) WRT the parameter $\theta(\theta=N v \Delta T \sin \gamma / r)$, yielding the 
following approximations

$$
\begin{aligned}
f_{1}= & \frac{4 N}{\theta^{2}}+\frac{4}{\theta^{2}}-\frac{5}{3}-\frac{2}{3 N}-N+\frac{31 \theta^{2} N}{180} \\
& +\frac{7 \theta^{2}}{18 N}-\frac{2 \theta^{2}}{45 N^{3}}+\frac{31 \theta^{2}}{60} \\
f_{2}= & \Delta T\left(\frac{N^{2}}{\theta}+\frac{N}{\theta}-7 \frac{N \theta}{12}-\frac{\theta}{3}-\theta \frac{N^{2}}{4}\right) \\
f_{3}= & \Delta T\left(-\frac{N}{2}-\frac{1}{3}-\frac{N^{2}}{6}-\frac{2}{45} \frac{\theta^{2}}{N^{2}}+2 \frac{\theta^{2}}{9}+\frac{\theta^{2} N}{4}+13 \frac{\theta^{2} N^{2}}{180}\right) \\
f_{4}= & (\Delta T)^{2}\left(\frac{N}{6}+\frac{N^{2}}{2}+\frac{N^{3}}{3}+\frac{\theta^{2}}{30 N}-\frac{\theta^{2} N}{8}-\frac{\theta^{2} N^{2}}{8}-\frac{\theta^{2} N^{3}}{30}\right) \\
f_{5}= & (\Delta T)^{2}\left(-\frac{N \theta}{6}-\frac{N^{2} \theta}{4}-\frac{N^{3} \theta}{12}\right) \\
f_{6}= & (\Delta T)^{2}\left(-\frac{\theta^{2}}{30 N}+\frac{\theta^{2} N}{8}+\frac{\theta^{2} N^{2}}{8}+\frac{\theta^{2} N^{3}}{30}\right)
\end{aligned}
$$

with

$$
\theta=\frac{N v \Delta T}{r} \sin \gamma \quad t_{m}=0
$$

The far source hypothesis $(\theta \ll 1)$ yields a major simplification of the above formula, so that retaining only the significant terms $(\Delta T=1)$, the following approximations are obtained:

$$
\begin{array}{ll}
f_{1} \simeq 4 \frac{\alpha^{2}}{N}, & f_{2} \simeq N \alpha, \quad f_{3} \simeq-\frac{N^{2}}{6} \\
f_{4} \simeq \frac{N^{3}}{3}, & f_{5} \simeq-\frac{N^{4}}{12 \alpha}, \quad f_{6} \simeq \frac{N^{5}}{30 \alpha^{2}}
\end{array}
$$

with

$$
\alpha \triangleq \frac{r}{v \sin \gamma} .
$$

The calculation of the diagonal terms of the inverse of the approximated FIM (eq. (41)) provides explicit expressions for the lower bounds of the variance of the kinematic parameters (i.e., the $X_{m, p}$ components), i.e.,

$$
\mid \begin{aligned}
& \operatorname{var}\left(\hat{r}_{y}\right) \simeq \frac{9}{4} \sigma_{\theta}^{2} N^{-1}(\text { bas })^{2} \\
& \operatorname{var}\left(\hat{v}_{x}\right) \simeq 57 \sigma_{\theta}^{2} N^{-3} r^{2} \\
& \operatorname{var}\left(\hat{v}_{y}\right) \simeq 180 \sigma_{\theta}^{2} N^{-3}\left(\frac{r}{\text { bas }}\right)^{2} r^{2}
\end{aligned}
$$

with bas $\triangleq N v \sin \gamma$ (= baseline).

It is interesting to compare the above results with those of the reference paper [1] even if they are obtained for a specific maneuver strategy of the ownship motion (special symmetric geometry). More precisely, and with the paper notations, the authors have obtained:

$$
\mid \begin{aligned}
& \operatorname{var}\left(\hat{v}_{x}\right) \simeq 12 \sigma_{\theta}^{2} N^{-3} r^{2} \\
& \operatorname{var}\left(\hat{v}_{y}\right) \simeq 144 \sigma_{\theta}^{2} N^{-3}\left(\frac{r}{\mathrm{bas}}\right)^{2} r^{2} .
\end{aligned}
$$

The two expressions (eqs. (42a), (b)) are thus rather similar to the classical one (eq. (43)). Let us consider now the above results.

1) $\operatorname{var}\left(\hat{r}_{y}\right)$ decreases with (bas) $)^{2}$, this result can be explained by the following facts: $r_{y}(0)$ is equal to $r$ (perfectly known) and, on another hand, as the baseline increases $r_{y}(t)$ differs from $r$.

2) $\operatorname{var}\left(\hat{v}_{y}\right)$ depends on the ratio range/baseline $(r /$ bas in (42)). In the extreme case where $\sin \gamma$ is zero then the variance of $\hat{v}_{y}$ becomes infinite.

3) $\operatorname{var}\left(\hat{v}_{x}\right)$ and $\operatorname{var}\left(\hat{v}_{y}\right)$ have common and classical factors $N^{-3}$ and $r^{2}$.

Similar calculations have been conducted for another value of $\theta_{c}\left(\theta_{c}=\pi / 4\right.$, see Fig. 3), yielding

$$
\begin{aligned}
& f_{1} \simeq 2 N, \quad f_{2} \simeq-\frac{N^{2}}{2}-\eta, \quad f_{3} \simeq \frac{N^{2}}{2}+2 \eta, \\
& f_{4} \simeq \frac{N^{3}}{6}-\frac{\varepsilon}{12}, \quad f_{5} \simeq-\frac{N^{3}}{6}+\frac{\theta \varepsilon}{30}, \quad f_{6} \simeq \frac{N^{3}}{3}+\frac{\varepsilon}{12}
\end{aligned}
$$

with:

$$
\eta=\frac{N^{2} \theta}{6} \quad \text { and } \quad \varepsilon=\theta N^{3} .
$$

Once again, the calculation of the diagonal terms of the inverse of the approximated FIM (eq. (44)) provides explicit approximations of the variances, more precisely one has:

$$
\begin{aligned}
& \operatorname{var}\left(\hat{r}_{y}\right) \simeq \frac{9}{8} \sigma_{\theta}^{2} r^{2} N^{-1} \\
& \operatorname{var}\left(\hat{v}_{x}\right) \simeq \operatorname{var}\left(\hat{v}_{y}\right) \simeq 90 \sigma_{\theta}^{2} N^{-3}\left(\frac{r}{\mathrm{bas}}\right)^{2} r^{2}
\end{aligned}
$$

with

$$
\text { bas }=N v \sin \left(\gamma-\theta_{c}\right) \quad \text { (baseline) } .
$$

A generalization of eq. 45 stands as follows:

$$
\begin{aligned}
& \operatorname{var}\left(\hat{r}_{y}\right) \simeq \frac{9}{4} N^{-1}\left(r \sin \theta \sigma_{\theta}\right)^{2} \\
& \operatorname{var}\left(\hat{v}_{x}\right) \simeq 180 N^{-3}\left(\frac{r}{\mathrm{bas}}\right)^{2}\left(r \sin \theta \sigma_{\theta}\right)^{2} \\
& \operatorname{var}\left(\hat{v}_{y}\right) \simeq 180 N^{-3}\left(\frac{r}{\mathrm{bas}}\right)^{2}\left(r \cos \theta \sigma_{\theta}\right)^{2}
\end{aligned}
$$

Let us consider now the above results.

1) $\operatorname{var}\left(\hat{v}_{x}\right)$ and $\operatorname{var}\left(\hat{v}_{y}\right)$ are approximately equal, they depend on $N$ and $r$ by the classical factors $\left(N^{-3}\right.$ and $\left.r^{2}\right)$, both depend on the parameter $(r /$ bas). Thus in the extreme case where $\sin \left(\gamma-\theta_{c}\right)$ is zero $\left(\gamma=\theta_{c}=\right.$ $\pi / 4)$ then the variances of $\hat{v}_{x}$ and $\hat{v}_{y}$ become infinite.

The validity of the formulas (42) and (45) has been studied by comparing them with the exact calculations (eq. (31)) for convenient scenari (long range source). The agreement is quite correct (relative error does not exceed a few percents).

Our attention is now focused on the spatialfrequency rate $(\dot{k})$ TMA performance. More precisely, 
the FIM matrix $G$ relative to the parameter vector $X_{m, p}$ (34) is directly deduced from (16), (18) giving

$G \triangleq \frac{1}{\sigma_{\dot{k}}^{2}}\left(\begin{array}{lll}g_{1} & g_{2} & g_{3} \\ g_{2} & g_{4} & g_{5} \\ g_{3} & g_{5} & g_{6}\end{array}\right)=\frac{1}{\sigma_{\dot{k}}^{2}} \sum_{i=1}^{L}\left(\nabla \dot{k}\left(X, t_{i}\right)\right)\left(\nabla \dot{k}\left(X, t_{i}\right)\right)^{\mathrm{T}}$

$\nabla \dot{k}\left(X, t_{i}\right) \triangleq\left(\frac{\partial \dot{k}\left(X, t_{i}\right)}{\partial r_{y}\left(t_{m}\right)}, \frac{\partial \dot{k}\left(X, t_{i}\right)}{\partial v_{x}}, \frac{\partial \dot{k}}{\partial v_{y}}\left(X, t_{i}\right)\right)^{\mathrm{T}}$

$L$ : number of available $\left(\hat{\dot{k}}_{i}\right)$.

Once again, a high order expansion of the various terms of the components $\left\{g_{i}\right\}$ WRT the parameter $\theta$ has been considered, yielding for the case $\theta_{c}=0$ (array broadside)

$$
\left\{\begin{array}{l}
g_{1}=\frac{4 L v^{2}}{r^{2} \theta^{2}}, \quad g_{2}=-\frac{2 L v_{y}}{r \theta} \\
g_{3}=\frac{N L v_{x} v_{y}}{r^{2} \theta}, \quad g_{4}=L \\
g_{5}=-\frac{1}{2} \frac{N L v_{x}}{r}, \quad g_{6}=\frac{N^{2} v_{x}^{2}}{r^{2}}\left(\frac{7 L^{2}-4}{12 L}\right) \\
\theta=\frac{N v \sin \gamma}{r} \quad\left(\theta_{c}=0\right)
\end{array}\right.
$$

where $L$ is number of $\left(\hat{\dot{k}}_{i}\right), N$ is total sample number.

From (47), it follows that $G$ is approximately a rank two matrix, leading us to consider the FIM $G^{\prime}$ restricted to the velocities estimation, i.e.,

$$
G^{\prime}=\left(\lambda^{2} \sigma_{\dot{k}}^{2} r^{2}\right)^{-1}\left(\begin{array}{ll}
g_{4} & g_{5} \\
g_{5} & g_{6}
\end{array}\right)
$$

yielding finally:

$$
\mid \begin{aligned}
& \operatorname{var}\left(\hat{v}_{x}\right) \simeq \lambda^{2} \sigma_{\dot{k}}^{2} r^{2}\left[\frac{7 L^{2}-4}{4 L\left(L^{2}-1\right)}\right] \\
& \operatorname{var}\left(\hat{v}_{y}\right) \simeq \lambda^{2} \sigma_{\dot{k}}^{2} r^{2}\left(\frac{r}{\mathrm{bas}}\right)^{2}\left(\frac{3 L}{L^{2}-1}\right) .
\end{aligned}
$$

Now, one has from (21):

$$
\sigma_{\dot{k}}^{2} \simeq \frac{18}{p^{3} \rho \pi^{2} d^{2}} \times \frac{1}{N_{1}^{3}}
$$

with $N_{1}$ being the number of samples associated with the estimation of a given $\hat{\dot{k}}_{i}, i=1, \ldots, L$, and

$$
N_{1} \times L=N
$$

so that

$$
\sigma_{\dot{k}}^{2} \simeq \frac{18}{p^{3} \rho \pi^{2} d^{2}} \frac{L^{3}}{N^{3}}
$$

with $N$ designing here the total number of samples.
Finally, the following approximations of $\operatorname{var}\left(\hat{v}_{x}\right)$ and $\operatorname{var}\left(\hat{v}_{y}\right)$ are obtained

$$
\begin{aligned}
& \operatorname{var}\left(\hat{v}_{x}\right) \simeq 3 \sigma_{\theta}^{2} r^{2} N^{-3}\left(\frac{L^{2}\left(7 L^{2}-4\right)}{4\left(L^{2}-1\right)}\right) \\
& \operatorname{var}\left(\hat{v}_{y}\right) \simeq 3 \sigma_{\theta}^{2} r^{2}\left(\frac{r}{\mathrm{bas}}\right)^{2} N^{-3}\left(\frac{3 L^{4}}{L^{2}-1}\right) .
\end{aligned}
$$

The above formulas (eq. (49)) are rather similar to the approximations of $\operatorname{var}\left(\hat{v}_{x}\right)$ and $\operatorname{var}\left(\hat{v}_{y}\right)$ obtained for the bearings-only analysis (eq. (42)) except for the constant terms and (obviously) the factor $L$.

First, note that according to (49) the factor $L$ must be chosen as little as possible. A direct application of (49) leads thus to choose $L$ equal to 2 . However, this choice must be mitigated by the adequacy of the linear approximation of $\dot{k}_{t}$ (eq. (19)). So that, in order to reduce the bias, a higher value of $L$ is generally necessary. This point is discussed in the analysis of the simulation results.

Actually, the values of $\operatorname{var}\left(\hat{v}_{x}\right)$ and $\operatorname{var}\left(\hat{v}_{y}\right)$ obtained by formulas (42) (bearings-only analysis) and (49) (spatial-frequency rate analysis) present a great similarity. Both depend similarly of the same geometric $(r, r /$ bas $)$ and statistical $\left(\sigma_{\theta}^{2}, N\right)$ factors. However, let us emphasize that the interest of spatial-frequency rate analysis relies on the availability of separated estimated values of $\left\{\dot{k}_{i}\right\}_{i=1}^{L}$. As seen later, the formula (49) confirms the interest of including the spatial-frequency rate in the TMA.

As previously, similar calculations have been conducted for $\theta_{c}=\pi / 4$, yielding the following approximations:

$$
\operatorname{var}\left(\hat{v}_{x}\right)=\operatorname{var}\left(\hat{v}_{y}\right) \simeq \lambda^{2} \sigma_{\dot{k}}^{2}\left(\frac{r}{\mathrm{bas}}\right)^{2} r^{2}\left(\frac{3 L}{L^{2}-1}\right) .
$$

As previously, the approximations of $\operatorname{var}\left(\hat{v}_{x}\right)$ and $\operatorname{var}\left(\hat{v}_{y}\right)$ are identical and depend upon the same geometric and statistic factors than for bearings-only TMA.

For the partial TMA problem, the reduced state is estimated conditionally to a given distance $r\left(t_{m}\right)$. Assume now that the following equality holds:

$$
r^{\prime}\left(t_{m}\right)=k r\left(t_{m}\right) \quad(k>0)
$$

then

$$
\left\{\begin{array}{l}
r_{x s}^{\prime}\left(t_{m}\right)=k r_{x s}\left(t_{m}\right)+(1+k) r_{x o}\left(t_{m}\right) \\
r_{y s}^{\prime}\left(t_{m}\right)=k r_{y s}\left(t_{m}\right)+(1-k) r_{y o}\left(t_{m}\right) \\
v_{x s}^{\prime}=k v_{x s}+(1-k) v_{x o} \\
v_{y s}^{\prime}=k v_{y s}+(1-k) v_{y o} .
\end{array}\right.
$$

Consequently, a relative error on $r\left(t_{m}\right)$ of a factor $k$ induces a relative error of the same factor for 
TABLE I

Ownship Trajectory

\begin{tabular}{|c|c|c|c|c|}
\hline \multicolumn{2}{|c|}{$\begin{array}{c}\text { Ownship } \\
\text { Position } t=0\end{array}$} & $\begin{array}{l}\text { Velocity Vector } \\
\text { Odd Legs }\end{array}$ & \multicolumn{2}{|c|}{$\begin{array}{l}\text { Velocity Vector } \\
\text { Even Legs }\end{array}$} \\
\hline \multicolumn{2}{|c|}{$v_{x 0}(0)=v_{y 0}(0)=0$} & $\begin{array}{l}v_{x 0}=3 \mathrm{~m} / \mathrm{s} \\
v_{y 0}=2 \mathrm{~m} / \mathrm{s}\end{array}$ & \multicolumn{2}{|c|}{$\begin{array}{c}v_{x 0}=-2 \mathrm{~m} / \mathrm{s} \\
v_{y 0}=3 \mathrm{~m} / \mathrm{s}\end{array}$} \\
\hline \multicolumn{5}{|c|}{$\begin{array}{c}\text { TABLE II } \\
\text { Target Trajectory }\end{array}$} \\
\hline & $r_{x s}$ & $r_{y s}$ & $v_{x s}$ & $v_{y s}$ \\
\hline$t=0$ & $15000 \mathrm{~m}$ & $35000 \mathrm{~m}$ & $2 \mathrm{~m} / \mathrm{s}$ & $5 \mathrm{~m} / \mathrm{s}$ \\
\hline$t=t_{m}$ & $21656 \mathrm{~m}$ & $51640 \mathrm{~m}$ & $2 \mathrm{~m} / \mathrm{s}$ & $5 \mathrm{~m} / \mathrm{s}$ \\
\hline
\end{tabular}

$\left(\operatorname{var}\left(r_{y s}\right)\right)^{1 / 2},\left(\operatorname{var}\left(v_{x s}\right)\right)^{1 / 2},\left(\operatorname{var}\left(v_{y s}\right)\right)^{1 / 2}$. Thus it is worthwhile (for practical utilization) to represent the statistical results by an abacus.

\section{SIMULATION RESULTS}

The previous results (especially Sections IV-VI) are now compared with simulation results. The comparisons are divided in two parts. The first one deals with the performance study for the observable case (nonuniform ownship motion, see Sections IV, V) while the second is devoted to the partially observable one (constant velocity vector of the ownship, see Section VI).

\section{A. TMA Performance (Observable Case)}

The purpose of the simulations is to illustrate the improvements of TMA induced by the inclusion of spatial-frequency rate estimates $(\hat{\dot{k}})$ in the TMA itself. For that aim, two scenarios are considered. The data are simulated by means of the variance formulas
TABLE III

Simulation Results and Comparison With Theoretical Bounds (Scenario 1)

\begin{tabular}{|c|c|c|c|c|}
\hline & $r_{x s}$ & $r_{y s}$ & $v_{x s}$ & $v_{y s}$ \\
\hline \multicolumn{5}{|c|}{ A. Bearings-Only TMA } \\
\hline $\begin{array}{l}\text { Empirical mean } \\
\text { (300 trials) }\end{array}$ & $21717 \mathrm{~m}$ & $51786 \mathrm{~m}$ & $2.1 \mathrm{~m} / \mathrm{s}$ & $5.03 \mathrm{~m} / \mathrm{s}$ \\
\hline $\begin{array}{l}\text { Empirical SD } 300 \\
\text { trials }\end{array}$ & $1001 \mathrm{~m}$ & $2321 \mathrm{~m}$ & $0.5 \mathrm{~m} / \mathrm{s}$ & $1.2 \mathrm{~m} / \mathrm{s}$ \\
\hline $\begin{array}{l}\text { Theoretical bounds } \\
\text { SD }\end{array}$ & $1076 \mathrm{~m}$ & $2491 \mathrm{~m}$ & $0.53 \mathrm{~m} / \mathrm{s}$ & $1.26 \mathrm{~m} / \mathrm{s}$ \\
\hline \multicolumn{5}{|c|}{ B. Bearings $+1 \hat{\dot{k}} /$ leg TMA } \\
\hline $\begin{array}{l}\text { Empirical mean } \\
\text { (300 trials) }\end{array}$ & $21655 \mathrm{~m}$ & $51637 \mathrm{~m}$ & $1.99 \mathrm{~m} / \mathrm{s}$ & $4.99 \mathrm{~m} / \mathrm{s}$ \\
\hline $\begin{array}{l}\text { Empirical SD ( } 300 \\
\text { trials) }\end{array}$ & $110 \mathrm{~m}$ & $255 \mathrm{~m}$ & $0.058 \mathrm{~m} / \mathrm{s}$ & $0.134 \mathrm{~m} / \mathrm{s}$ \\
\hline $\begin{array}{l}\text { Theoretical bounds } \\
\text { SD }\end{array}$ & $106 \mathrm{~m}$ & $239 \mathrm{~m}$ & $0.055 \mathrm{~m} / \mathrm{s}$ & $0.124 \mathrm{~m} / \mathrm{s}$ \\
\hline \multicolumn{5}{|c|}{ C. Bearings $+3 \hat{\dot{k}} / \operatorname{leg}$ TMA } \\
\hline $\begin{array}{l}\text { Empirical mean } \\
\text { (300 trials) }\end{array}$ & $21637 \mathrm{~m}$ & $51600 \mathrm{~m}$ & $1.99 \mathrm{~m} / \mathrm{s}$ & $4.97 \mathrm{~m} / \mathrm{s}$ \\
\hline $\begin{array}{l}\text { Empirical SD ( } 300 \\
\text { trials) }\end{array}$ & $286 \mathrm{~m}$ & $656 \mathrm{~m}$ & $0.14 \mathrm{~m} / \mathrm{s}$ & $0.32 \mathrm{~m} / \mathrm{s}$ \\
\hline $\begin{array}{l}\text { Theoretical bounds } \\
\text { SD }\end{array}$ & $278 \mathrm{~m}$ & $636 \mathrm{~m}$ & $0.13 \mathrm{~m} / \mathrm{s}$ & $0.31 \mathrm{~m} / \mathrm{s}$ \\
\hline
\end{tabular}

relative to $\hat{k}_{t}$ and $\hat{\dot{k}}_{\ell}$ (more precisely (6) and (21)). The general simulation parameters are presented here:

1) linear array of sensors (32 equispaced sensors, $d=2 m$ ), 2) signal-to-noise ratio: $\rho=-10 \mathrm{~dB}, 3$ ) total number of snapshots: $N=2000$, each bearing estimate corresponds to 10 snapshots.

The parameters of the two scenarios are detailed below in Tables I-III.

\section{Scenario 1}

number of estimated bearings: (200).

number of legs: $L=4$.

total simulation duration: $T=3328 \mathrm{~s}$ referenced WRT $t_{m}=3328 \mathrm{~s}$ ).

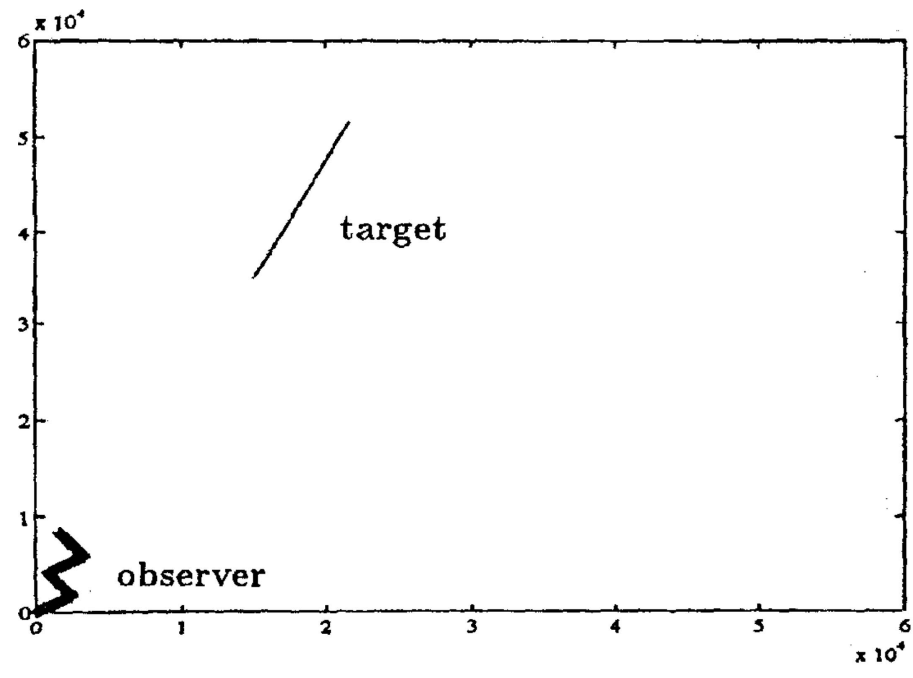

Fig. 4. Source-observer geometry of scenario 1 . 
TABLE IV

Ownship Trajectory

\begin{tabular}{ccc}
\hline \hline $\begin{array}{c}\text { Ownship } \\
\text { Position } t=0\end{array}$ & $\begin{array}{c}\text { Velocity Vector } \\
\text { Odd Legs }\end{array}$ & $\begin{array}{c}\text { Velocity Vector } \\
\text { Even Legs }\end{array}$ \\
\hline \multirow{2}{*}{$r_{x 0}(0)=r_{y 0}(0)=0$} & $v_{x 0}=5 \mathrm{~m} / \mathrm{s}$ & $v_{x 0}=0 \mathrm{~m} / \mathrm{s}$ \\
& $v_{y 0}=0 \mathrm{~m} / \mathrm{s}$ & $v_{y 0}=5 \mathrm{~m} / \mathrm{s}$ \\
\hline
\end{tabular}

TABLE V

Target Trajectory

\begin{tabular}{crccc}
\hline \hline & $r_{x s}$ & $r_{y s}$ & $v_{x s}$ & $v_{y s}$ \\
\hline$t=0$ & $5000 \mathrm{~m}$ & $20000 \mathrm{~m}$ & $6 \mathrm{~m} / \mathrm{s}$ & $3 \mathrm{~m} / \mathrm{s}$ \\
$t=t_{m}$ & $14812 \mathrm{~m}$ & $24806 \mathrm{~m}$ & $6 \mathrm{~m} / \mathrm{s}$ & $3 \mathrm{~m} / \mathrm{s}$ \\
\hline
\end{tabular}

The geometry of this TMA problem is depicted in Fig. 4. The target motion parameters are then estimated by means of the iterative algorithm (Gauss-Newton) described in Section IV. This algorithm does not suffer from any problem of convergence (in the unique source case). The whole scenario is repeated 300 times allowing us to compute empirical estimates of the variances of source motion parameter estimates and to compare them with the calculated bounds (Section V). The results are summarized in Table III.

Scenario 2 (see Table IV-VI)

number of estimated bearings: (200).

number of estimated values $\hat{\dot{k}} / \mathrm{leg}: 4$.

total simulation duration: $T=1602 \mathrm{~s}\left(t_{m}=1602 \mathrm{~s}\right)$.

The geometry of this TMA problem is depicted in

Fig. 5. The results are summarized in Table VI.

These simulation results require some comments.
TABLE VI

Simulation Results and Comparison With Theoretical Bounds (Scenario 2)

\begin{tabular}{|c|c|c|c|c|}
\hline & $r_{x s}$ & $r_{y s}$ & $v_{x s}$ & $v_{y s}$ \\
\hline \multicolumn{5}{|c|}{ A. Bearings-Only TMA } \\
\hline $\begin{array}{l}\text { Empirical mean } \\
(300 \text { trials })\end{array}$ & $14569 \mathrm{~m}$ & $24724 \mathrm{~m}$ & $5.97 \mathrm{~m} / \mathrm{s}$ & $2.91 \mathrm{~m} / \mathrm{s}$ \\
\hline $\begin{array}{l}\text { Empirical SD } 300 \\
\text { trials }\end{array}$ & $246 \mathrm{~m}$ & $588 \mathrm{~m}$ & $0.2 \mathrm{~m} / \mathrm{s}$ & $0.61 \mathrm{~m} / \mathrm{s}$ \\
\hline $\begin{array}{l}\text { Theoretical bounds } \\
\text { SD }\end{array}$ & $275 \mathrm{~m}$ & $660 \mathrm{~m}$ & $0.23 \mathrm{~m} / \mathrm{s}$ & $0.67 \mathrm{~m} / \mathrm{s}$ \\
\hline \multicolumn{5}{|c|}{ B. Bearings $+\hat{\dot{k}}(3$ legs $)$} \\
\hline $\begin{array}{l}\text { Empirical mean } \\
\text { Empirical SD } \\
\text { Theoretical bounds }\end{array}$ & $\begin{array}{r}14600 \mathrm{~m} \\
31 \mathrm{~m} \\
29 \mathrm{~m}\end{array}$ & $\begin{array}{r}24802 \mathrm{~m} \\
79 \mathrm{~m} \\
70 \mathrm{~m}\end{array}$ & $\begin{array}{l}6.00 \mathrm{~m} / \mathrm{s} \\
0.031 \mathrm{~m} / \mathrm{s} \\
0.026 \mathrm{~m} / \mathrm{s}\end{array}$ & $\begin{array}{l}3.00 \mathrm{~m} / \mathrm{s} \\
0.095 \mathrm{~m} / \mathrm{s} \\
0.081 \mathrm{~m} / \mathrm{s}\end{array}$ \\
\hline \multicolumn{5}{|c|}{ C. Bearings $+\hat{\dot{k}}(4$ legs $)$} \\
\hline $\begin{array}{l}\text { Empirical mean } \\
\text { Empirical SD } \\
\text { Theoretical bounds } \\
\text { SD }\end{array}$ & $\begin{array}{r}14611 \mathrm{~m} \\
40 \mathrm{~m} \\
26 \mathrm{~m}\end{array}$ & $\begin{array}{r}24793 \mathrm{~m} \\
77 \mathrm{~m} \\
52 \mathrm{~m}\end{array}$ & $\begin{array}{l}6.01 \mathrm{~m} / \mathrm{s} \\
0.026 \mathrm{~m} / \mathrm{s} \\
0.018 \mathrm{~m} / \mathrm{s}\end{array}$ & $\begin{array}{ll}2.99 \mathrm{~m} / \mathrm{s} \\
0.11 \mathrm{~m} / \mathrm{s} \\
0.053 \mathrm{~m} / \mathrm{s}\end{array}$ \\
\hline
\end{tabular}

1) The quality of the target parameter estimates is greatly improved by the utilization of the estimated spatial-frequency rates $\hat{\dot{k}}$. This is mainly due to the value of $\sigma_{\dot{k}}^{2}$ (eq. (21)), corresponding to long integration times.

2) It seems preferable to use a reduced number of $\hat{\dot{k}}$ per leg (e.g., one $\hat{\dot{k}} /$ leg), which confirms the theoretical results of Section VI.

3) In scenario 2 the standard deviation (SD) of the velocity estimates decreases with the number of legs (all other parameters being fixed).

4) Practically, the improvements relative to the estimation of the components of the (target) stage vector (SD) are comprised between 3 and 10. They decrease with the number of $\hat{\dot{k}}$ per leg and with the inverse of the distance observer-target.

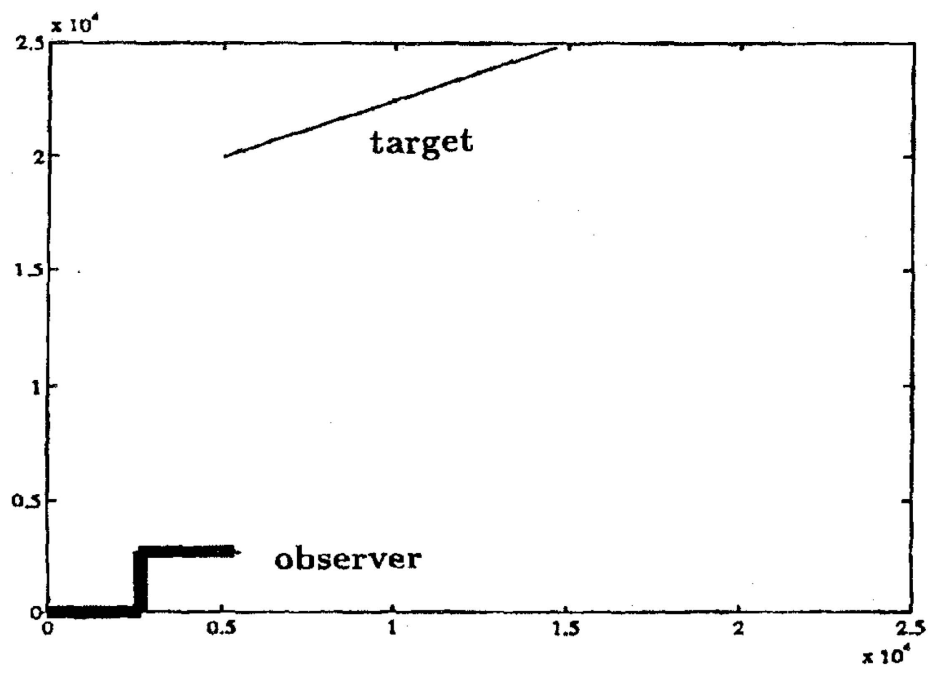

Fig. 5. Source-observer geometry of scenario 2 . 
TABLE VII

TMA Parameters (Scenario 3)

\begin{tabular}{|c|c|c|c|c|c|c|}
\hline & & $x s(t)$ & $r_{y s}(t)$ & $r(t)$ & $v_{x s}$ & $v_{y s}$ \\
\hline \multirow{3}{*}{$\begin{array}{c}t=0 \\
t=t_{m}\end{array}$} & & $000 \mathrm{~m}$ & $10000 \mathrm{~m} 5$ & $50999 \mathrm{~m}$ & $-3 \mathrm{~m} / \mathrm{s}$ & $5 \mathrm{~m} / \mathrm{s}$ \\
\hline & 452 & $200 \mathrm{~m}$ & $18000 \mathrm{~m} 4$ & $41326 \mathrm{~m}$ & $-3 \mathrm{~m} / \mathrm{s}$ & $5 \mathrm{~m} / \mathrm{s}$ \\
\hline & & $\bar{\theta}(\mathrm{deg}$ & $\sigma_{\theta}(\mathrm{deg})$ & ) & & $\sigma_{k}$ \\
\hline \multirow{2}{*}{\multicolumn{2}{|c|}{$\begin{array}{l}\rho=-10 \mathrm{~dB} \\
\rho=-20 \mathrm{~dB}\end{array}$}} & 71.9 & 0.96 & \multirow{2}{*}{\multicolumn{3}{|c|}{$\begin{array}{ll}-1.25 \times 10^{-5} & 2.6 \times 10^{-6} \\
-1.25 \times 10^{-5} & 1.45 \times 10^{-5}\end{array}$}} \\
\hline & & 71.9 & 5.41 & & & \\
\hline
\end{tabular}

\section{B. TMA Performance, Partially Observable Case}

As previously, these simulations deal with the study of the effects of including $\hat{\dot{k}}$ in the TMA. The general simulation parameters are presented here:

1) linear array of sensors (32 equispaced sensors),

2) total duration of the TMA scenario (1600 s),

3 ) total number of snapshots $N=1200$, number of estimated bearings: 120 (each bearing estimated on 10 snapshots), 4) number of estimated $\hat{\dot{k}}: 4$ (each estimated on 300 snapshots), 5) initial position of the ownship $\left(r_{x o}=r_{y o}=0 \mathrm{~m}\right)$, ownship velocity vector $\left(v_{x o}=5 \mathrm{~m} / \mathrm{s}, v_{y o}=0 \mathrm{~m} / \mathrm{s}\right)$.

The parameters of the two scenarios are now presented.

Scenario 3: This scenario is rather critical since the target is situated in the vicinity of the array axis (endfire). Two values of signal to noise ratio are considered and shown in Table VII.

The target-ownship geometry is depicted in Fig. $6, \bar{\theta}$ and $\bar{k}$ stand for the averaged (deterministic) values of $\theta$ and $\dot{k}$. Then the results are summarized in Table VIII.
TABLE VIII

Simulation Results and Comparison With Theoretical Bounds (Scenario 3). Range $r$ Replaced by Its Exact Value

\begin{tabular}{|c|c|c|c|}
\hline & $r_{y s}\left(t_{m}\right)$ & $v_{x s}$ & $v_{y s}$ \\
\hline A. & \multicolumn{2}{|c|}{ Bearings-Only TMA } & \\
\hline Mean 300 trials & $17989 \mathrm{~m}$ & $-3.1 \mathrm{~m} / \mathrm{s}$ & $4.94 \mathrm{~m} / \mathrm{s}$ \\
\hline Empirical SD & $133 \mathrm{~m}$ & $2.44 \mathrm{~m} / \mathrm{s}$ & $0.57 \mathrm{~m} / \mathrm{s}$ \\
\hline Theoretical bound SD & $133 \mathrm{~m}$ & $2.42 \mathrm{~m} / \mathrm{s}$ & $0.57 \mathrm{~m} / \mathrm{s}$ \\
\hline \multicolumn{4}{|c|}{ B. Bearings $+\dot{k}$ TMA } \\
\hline Mean 300 trials & $17999 \mathrm{~m}$ & $-2.99 \mathrm{~m} / \mathrm{s}$ & $5.00 \mathrm{~m} / \mathrm{s}$ \\
\hline Empirical SD & $44 \mathrm{~m}$ & $0.52 \mathrm{~m} / \mathrm{s}$ & $0.16 \mathrm{~m} / \mathrm{s}$ \\
\hline Theoretical bound SD & $48 \mathrm{~m}$ & $0.50 \mathrm{~m} / \mathrm{s}$ & $0.16 \mathrm{~m} / \mathrm{s}$ \\
\hline C. & \multicolumn{2}{|c|}{ Bearings-Only TMA } & \\
\hline Mean 300 trials & $18065 \mathrm{~m}$ & $-5.53 \mathrm{~m} / \mathrm{s}$ & $4.32 \mathrm{~m} / \mathrm{s}$ \\
\hline Empirical SD & $753 \mathrm{~m}$ & $15.69 \mathrm{~m} / \mathrm{s}$ & $3.9 \mathrm{~m} / \mathrm{s}$ \\
\hline Theoretical bound SD & $749 \mathrm{~m}$ & $13.59 \mathrm{~m} / \mathrm{s}$ & $3.23 \mathrm{~m} / \mathrm{s}$ \\
\hline \multicolumn{4}{|c|}{ D. Bearings $+\dot{k}$ TMA } \\
\hline Mean 300 trials & $18008 \mathrm{~m}$ & $-3.4 \mathrm{~m} / \mathrm{s}$ & $4.7 \mathrm{~m} / \mathrm{s}$ \\
\hline Empirical SD & $324 \mathrm{~m}$ & $2.7 \mathrm{~m} / \mathrm{s}$ & $1.14 \mathrm{~m} / \mathrm{s}$ \\
\hline Theoretical bound SD & $271 \mathrm{~m}$ & $2.8 \mathrm{~m} / \mathrm{s}$ & $0.91 \mathrm{~m} / \mathrm{s}$ \\
\hline
\end{tabular}

Note: $\rho=-10 \mathrm{~dB}$ in $\mathrm{A}$ and B. $\rho=-20 \mathrm{~dB}$ in $\mathrm{C}$ and $\mathrm{D}$.

Scenario 4: An easier scenario is now considered. The source trajectory parameters are detailed in Table IX.

The target-ownship geometry is depicted in Fig. 7 and the results are summarized in Table X.

As previously, the quality of the source trajectory parameter estimate is greatly improved by the spatial-frequency rates $\hat{\dot{k}}$. This is especially true for a weak source $(\rho=-20 \mathrm{~dB})$, since in this case the SD of bearings-only TMA relative to velocity is very important (see Tables VIII and X). Then, the

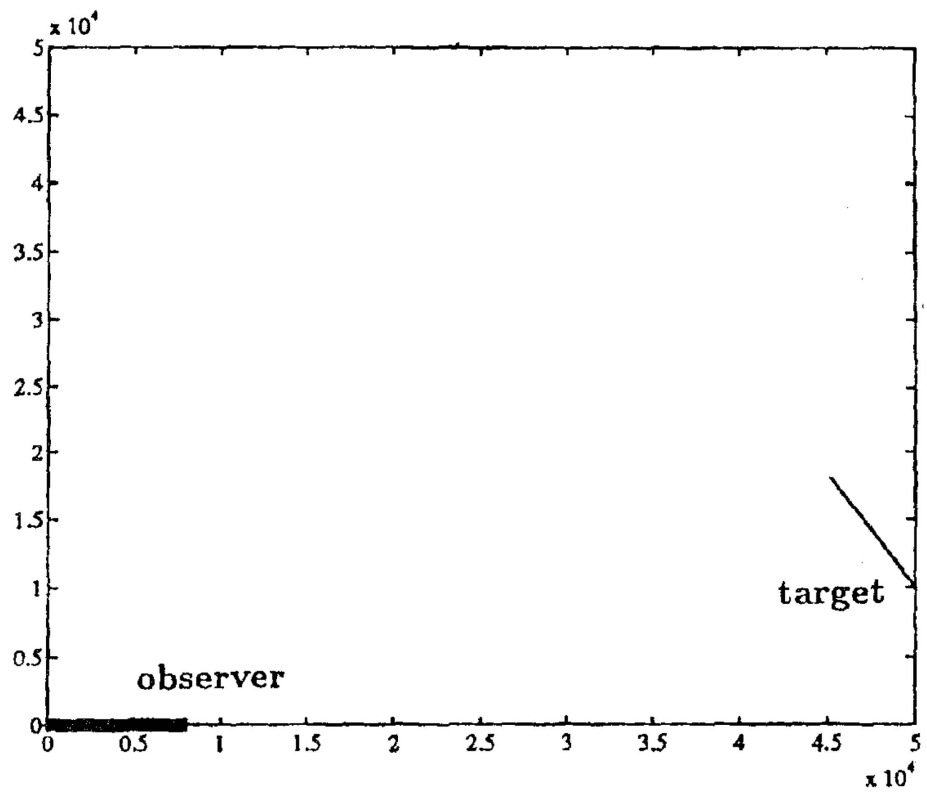

Fig. 6. Source-observer geometry of scenario 3 . 
TABLE IX

TMA Parameters (Scenario 4)

\begin{tabular}{|c|c|c|c|c|c|c|}
\hline & & $x s(t)$ & $r_{y s}(t)$ & $r(t)$ & $v_{x s}$ & $v_{y s}$ \\
\hline \multirow{3}{*}{$\begin{array}{c}t=0 \\
t=t_{m}\end{array}$} & \multirow{2}{*}{\multicolumn{2}{|c|}{$\begin{array}{l}30000 \mathrm{~m} \\
36400 \mathrm{~m}\end{array}$}} & \multirow{2}{*}{$\begin{array}{l}30000 \mathrm{~m} \\
23600 \mathrm{~m}\end{array}$} & $42426 \mathrm{~m}$ & $4 \mathrm{~m} / \mathrm{s}$ & $-4 \mathrm{~m} / \mathrm{s}$ \\
\hline & & & & $36926 \mathrm{~m}$ & $4 \mathrm{~m} / \mathrm{s}$ & $-4 \mathrm{~m} / \mathrm{s}$ \\
\hline & \multicolumn{2}{|r|}{$\bar{\theta}(\operatorname{deg})$} & $\sigma_{\theta}(\mathrm{deg}$ & ) & & $\sigma_{k}$ \\
\hline \multirow{2}{*}{\multicolumn{2}{|c|}{$\begin{array}{l}\rho=-10 \mathrm{~dB} \\
\rho=-20 \mathrm{~dB}\end{array}$}} & 47,5 & 0.42 & \multirow{2}{*}{\multicolumn{2}{|c|}{$\begin{array}{l}-9.7 \times 10^{-6} \\
-9.7 \times 10^{-6}\end{array}$}} & $2.6 \times 10^{-6}$ \\
\hline & & 47,5 & 2.35 & & & $1.45 \times 10^{-5}$ \\
\hline
\end{tabular}

bearings $+\dot{k}$ TMA reduce dramatically the SD of source velocity components.

\section{TOWARDS MULTIPLE SOURCE TMA}

In numerous situations the quality of the source tracks is very questionable. This occurs frequently in practical situations like sources crossing, change of sources' level, etc. Even with a good step of data association, the statistical quality of the track may be seriously degraded. A way to overcome this problem consists in merging data association and source tracking $[20,21]$ or in considering multiple sources TMA [22]. However, multiple sources TMA is frequently considered after source tracking and data association steps [23]. Furthermore, the (nonlinear) estimation of the multiple source's state requires an iterative optimization of the likelihood functional. The optimization can thus be rather time consuming but, overall, can suffer from numerous local minima [21, $23]$. Even if it is (theoretically) possible to overcome this problem by using extensive computations (e.g., annealing method), the convergence of the whole
TABLE $\mathrm{X}$

Simulation Results and Comparison With Theoretical Bounds (Scenario 4). Range $r$ Replaced by Its Exact Value

\begin{tabular}{|c|c|c|c|}
\hline & $r_{y s}\left(t_{m}\right)$ & $v_{x s}$ & $v_{y s}$ \\
\hline A. & \multicolumn{2}{|c|}{ Bearings-Only TMA } & \\
\hline Mean 300 trials & $23602 \mathrm{~m}$ & $4.14 \mathrm{~m} / \mathrm{s}$ & $-3.85 \mathrm{~m} / \mathrm{s}$ \\
\hline Empirical SD & $54 \mathrm{~m}$ & $1.76 \mathrm{~m} / \mathrm{s}$ & $1.75 \mathrm{~m} / \mathrm{s}$ \\
\hline Theoretical bound SD & $60 \mathrm{~m}$ & $1.83 \mathrm{~m} / \mathrm{s}$ & $1.83 \mathrm{~m} / \mathrm{s}$ \\
\hline \multicolumn{4}{|c|}{ B. Bearings $+\dot{k}$ TMA } \\
\hline Mean 300 trials & $23618 \mathrm{~m}$ & $4.38 \mathrm{~m} / \mathrm{s}$ & $-3.58 \mathrm{~m} / \mathrm{s}$ \\
\hline Empirical SD & $30 \mathrm{~m}$ & $0.44 \mathrm{~m} / \mathrm{s}$ & $0.46 \mathrm{~m} / \mathrm{s}$ \\
\hline Theoretical bound SD & $26 \mathrm{~m}$ & $0.33 \mathrm{~m} / \mathrm{s}$ & $0.33 \mathrm{~m} / \mathrm{s}$ \\
\hline C. & \multicolumn{2}{|c|}{ Bearings-Only TMA } & \\
\hline Mean 300 trials & $23591 \mathrm{~m}$ & $1.83 \mathrm{~m} / \mathrm{s}$ & $-6.07 \mathrm{~m} / \mathrm{s}$ \\
\hline Empirical SD & $350 \mathrm{~m}$ & $12.90 \mathrm{~m} / \mathrm{s}$ & $12.80 \mathrm{~m} / \mathrm{s}$ \\
\hline Theoretical bound SD & $340 \mathrm{~m}$ & $10.26 \mathrm{~m} / \mathrm{s}$ & $10.26 \mathrm{~m} / \mathrm{s}$ \\
\hline \multicolumn{4}{|c|}{ D. Bearings $+\dot{k}$ TMA } \\
\hline Mean 300 trials & $23629 \mathrm{~m}$ & $4.11 \mathrm{~m} / \mathrm{s}$ & $-3.88 \mathrm{~m} / \mathrm{s}$ \\
\hline Empirical SD & $211 \mathrm{~m}$ & $2.12 \mathrm{~m} / \mathrm{s}$ & $2.11 \mathrm{~m} / \mathrm{s}$ \\
\hline Theoretical bound SD & $144 \mathrm{~m}$ & $1.85 \mathrm{~m} / \mathrm{s}$ & $1.85 \mathrm{~m} / \mathrm{s}$ \\
\hline
\end{tabular}

Note: $\rho=-10 \mathrm{~dB}$ in $\mathrm{A}$ and B. $\rho=-20 \mathrm{~dB}$ in $\mathrm{C}$ and $\mathrm{D}$.

process is not ensured. A radically new way for multiple sources TMA is now presented.

It relies upon the properties of the 2D state space model of the spatio-temporal data derived in Section III, itself based on the simplified model (eq. (10)) of the spatial frequency of a moving source. The advantages for multiple source TMA are presented here.

1) The 2D state space model of the spatiotemporal data is linear.

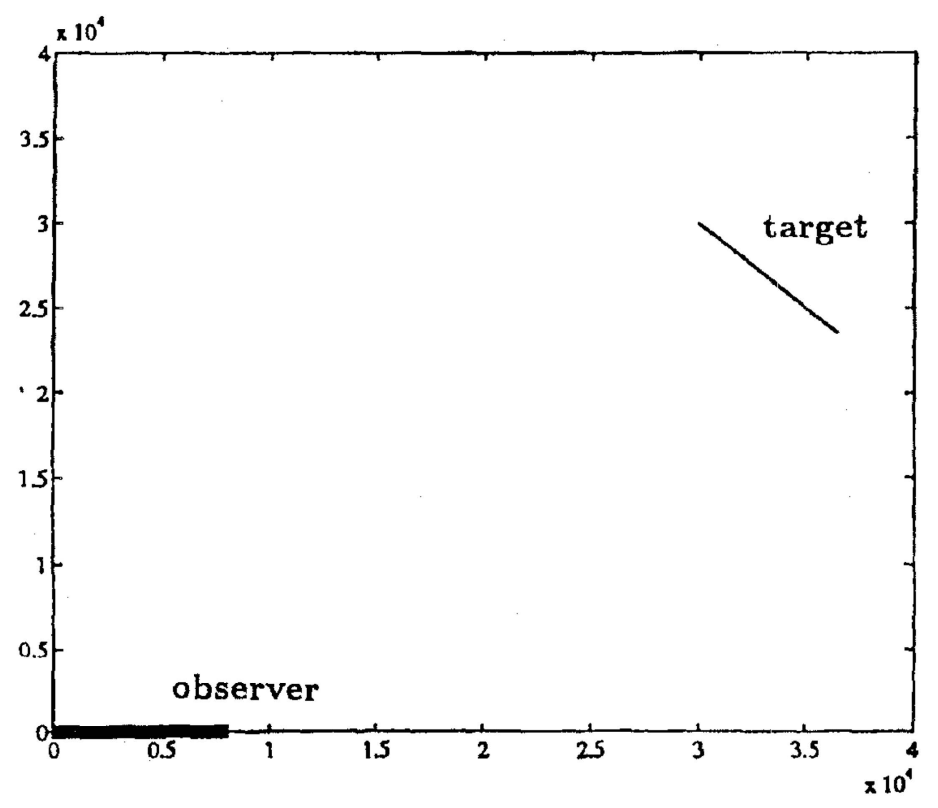

Fig. 7. Source-observer geometry of scenario 4 . 
2) The parameters $\left\{k_{0, i}\right\}_{i=1}^{s}$ and $\left\{\dot{k}_{i}\right\}_{i=1}^{s}$ may be separately and directly estimated by a noniterative and simple procedure.

3) The association of the various $\left\{\hat{\dot{k}}_{i, t}\right\}$ is greatly simplified by their closeness.

The first point means that the (nonlinear) state estimation, classical in TMA [1], is replaced by an estimation of the transition matrix $F_{1}$. More precisely, the problem becomes a realization problem [24, 25]. Furthermore, thanks to the special structure of the 2D state space model (26), the transition matrix $F_{1}$ may be estimated apart from $F_{0}$ and, more important, without any a priori knowledge of $F_{0}$ (the initial spatial frequencies). This last point is fundamental since it avoids the basic problem of sources interference [7] and allows us to derive direct (noniterative) methods for estimating $F_{1}$, avoiding, thus, the local minima problems. Furthermore, these methods can be extended to the whole 2D array of spatio-temporal data (large integration time). Note that the changes of sources' level do not affect basically the performance of these methods (except for the estimation noise level).

The parameters $\left\{\dot{k}_{i}\right\}_{i=1}^{s}$ being estimated, the data association is greatly simplified and these parameters can be incorporated in the extended TMA algorithm presented previously (Section IV). Let us now present a multidimensional estimation of the parameters $\dot{k}_{j}$. This approach is multidimensional and multiscale and relies heavily on an interpolation procedure presented below.

Using (26), the following interpolation formula is valid for the state vector of the "STS" $m$. The STS (spatio-temporal sequence) $m$ designs here the temporal sequence $\{y(t, m)\}_{t}$ (the spatial index $m$ is fixed).

$$
X\left(t+\frac{m_{0}}{m}, m\right)=F_{1}^{m_{0}} X(t, m) .
$$

Actually, this formula simply results from the expression of the exact spatio-temporal covariances $r_{m}(t)(25):$

$$
r_{m}(t)=\sum_{j=1}^{s} \sigma_{j}^{2} \exp \left[2 i \pi d m\left(k_{j, 0}+t \dot{k}_{j}\right)\right] .
$$

Therefore, according to (26) and (52), the interpolated data $\tilde{z}(t, m)$ corresponding to state space model outputs (26) without noise, satisfy:

$$
\begin{aligned}
\tilde{z}\left(t+j \frac{m_{0}}{m}, m\right) & =h^{*} X\left(t+j \frac{m_{0}}{m}, m\right) \\
& =h^{*} F_{1}^{j m_{0}} X(t, m) .
\end{aligned}
$$

It is thus possible to consider the same power of the transition matrix $F_{1}$ (i.e., $F_{1}^{m_{0}}$ ) by interpolating the various STS.
Let $\tilde{\mathcal{H}}_{t, m_{0}, c}$ be the matrix built with the interpolated $\tilde{y}$ data on the various STS, i.e.,

$$
\begin{aligned}
& \tilde{\mathcal{H}}_{t, m_{0}, c}= \\
& \left(\begin{array}{cccc}
y\left(t, m_{0}\right) & y\left(t, m_{0}+1\right) & \ldots & y\left(t, m_{0}+c\right) \\
y\left(t+1, m_{0}\right) & \tilde{y}\left(t+\tau_{1}, m_{0}+1\right) & \ldots & \tilde{y}\left(t+\tau_{c}, m_{0}+c\right) \\
\vdots & \vdots & \vdots & \\
y\left(t+r, m_{0}\right) & \tilde{y}\left(t+r \tau_{1}, m_{0}+1\right) & \cdots & \tilde{y}\left(t+r \tau_{c}, m_{0}+c\right)
\end{array}\right)
\end{aligned}
$$

with

$$
\tau_{1} \triangleq \frac{m_{0}}{m_{0}+1}, \tau_{2} \triangleq \frac{m_{0}}{m_{0}+2}, \ldots, \tau_{c} \triangleq \frac{m_{0}}{m_{0}+c} .
$$

The scalars $\tau_{1}, \tau_{2}, \ldots, \tau_{c}$ represent the compression (of time) factors relative to the STS $m_{0}+1, \ldots, m_{0}+c$. In this case, the reference STS corresponds to the spatial index $m_{0}$. Obviously the choice of this reference index is arbitrary.

Now, without consideration of the estimation noise (relative to $y$ ) the following factorization holds:

$$
\begin{aligned}
\tilde{\mathcal{H}}_{t, m_{0}, c}= & \left(\begin{array}{c}
h^{*} \\
h^{*} F_{1}^{m_{0}} \\
h^{*} F_{1}^{2 m_{0}} \\
\vdots \\
h^{*} F_{1}^{r m_{0}}
\end{array}\right) \\
& \times\left(X\left(t, m_{0}\right), X\left(t, m_{0}+1\right), \ldots, X\left(t, m_{0}+c\right)\right) \\
= & \mathcal{O} \mathcal{X}_{t},
\end{aligned}
$$

with

$$
\begin{aligned}
\mathcal{X}_{t} & \triangleq \\
\mathcal{O} & : \text { observability matrix }
\end{aligned}
$$

A direct consequence of (55) is the following rank property $[24,26]$ :

$$
\operatorname{rank}\left(\tilde{\mathcal{H}}_{t, m_{0}, c}\right)=\operatorname{rank}(\mathcal{O})=\operatorname{dim}(X) .
$$

Practically the estimated observability matrix $\hat{\mathcal{O}}$ is obtained from $\tilde{\mathcal{H}}_{t, m_{0}, c}$ by means of a singular value decomposition (SVD) [27] and an estimated matrix $\hat{F}_{1}$ is deduced from $\hat{\mathcal{O}}$ by using the classical shift property, i.e. [24],

$$
\mathcal{O}^{\uparrow} F_{1}^{m_{0}}=\mathcal{O}^{\downarrow}
$$

with

$$
\mathcal{O}^{\uparrow}=\left(\begin{array}{l}
h^{*} \\
h^{*} F_{1}^{m_{0}} \\
\vdots \\
h^{*} F_{1}^{(r-1) m_{0}}
\end{array}\right) \quad \mathcal{O}^{\downarrow}=\left(\begin{array}{c}
h^{*} F_{1}^{m_{0}} \\
h^{*} F_{1}^{2 m_{0}} \\
\vdots \\
h^{*} F_{1}^{r m_{0}}
\end{array}\right) .
$$


The previous method seems to be promising thanks to its simplicity. However, the main limitation comes from the estimation noise $w(t, m)$ (defined in (26)) of the spatio-temporal data $y(t, m)$. Fortunately, this noise has a particular and useful property: it is spatially correlated and its correlation matrix is proportional to a sum of the sources' covariance matrices.

Actually, the correlation matrix of the estimation noise takes the following form in the unique source case:

$$
\mathbb{E}\left[w\left(t, m_{1}\right) w^{*}\left(t, m_{2}\right)\right]=\rho^{2} \exp \left(-2 i \pi d\left(m_{2}-m_{1}\right) k_{t}\right)
$$

where $m_{1} \neq m_{2}, \rho$ : signal-to-noise ratio.

For the two sources case, the exact expression of $\operatorname{cov}\left(w\left(t, m_{1}\right) w^{*}\left(t, m_{2}\right)\right)$ is rather complicated [7] but its asymptotic value (sensor number $p$ great) is quite interesting since:

$$
\begin{aligned}
\lim _{p \rightarrow \infty} \mathbb{E} & {\left[w\left(t, m_{1}\right) w^{*}\left(t, m_{2}\right)\right] } \\
= & \rho_{1}^{2} \exp \left(-2 i \pi d\left(m_{2}-m_{1}\right) k_{1, t}\right) \\
& +\rho_{2}^{2} \exp \left(-2 i \pi d\left(m_{2}-m_{1}\right) k_{2, t}\right)
\end{aligned}
$$

( $\rho_{1}$ and $\rho_{2} s / n$ ratios of the two sources).

Consequently, the covariance matrix of the estimation noise $\{w(t, m)\}_{m}$ tends towards a matrix proportional to the sum of the covariance matrices of the strong sources. Therefore, the estimation noise $w(t, m)$ which is a limitating factor for multiple sources TMA, may be seriously reduced by applying a spatial filter to the data. The simpler one is the beamforming [3].

In order to detect (track) a weak moving source, it is worthwhile to consider a set of beams $\left(k_{0}, k_{1}, \ldots, k_{\ell}\right)$ whose spatial frequencies $k_{i}$ are chosen ( $3 \mathrm{~dB}$ covering) in order to "isolate" a sector as described in Fig. 8. The formalism of the spatio-temporal analysis is similar to the previous one (eqs. (52)-(57)) and is, thus, briefly described below [7]. Let us, first, define the vectors $Y_{t}, \tilde{Y}_{t+1}, \tilde{Y}_{t+2} \ldots$ :

$$
\begin{aligned}
& Y_{t} \triangleq\left(y\left(t, m_{0}\right), y\left(t, m_{0}+1\right), \ldots, y(t, m)\right)^{\mathrm{T}} \\
& \tilde{Y}_{t+1} \triangleq\left(y\left(t+1, m_{0}\right), \tilde{y}\left(t+\tau_{1}, m_{0}+1\right), \ldots\right)^{\mathrm{T}} . \\
& \text {... }
\end{aligned}
$$

Note that the vectors $Y_{t}, \tilde{Y}_{t+1}, \tilde{Y}_{t+2}$ are anything but the rows of the matrix $\tilde{\mathcal{H}}_{t, m_{0}, c}$ and therefore (eq. (53)) the following property holds (without consideration of the estimation noise):

$$
\begin{aligned}
D_{k_{0}}^{*} Y_{t} & =h^{*}\left(\sum_{j=m_{0}}^{m} \exp \left(2 i \pi d j k_{0}\right) X(t, j)\right) \\
D_{k_{0}}^{*} \tilde{Y}_{t+1} & =h^{*} F_{1}\left(\sum_{j=m_{0}}^{m} \exp \left(2 i \pi d j k_{0}\right) X(t, j)\right) .
\end{aligned}
$$

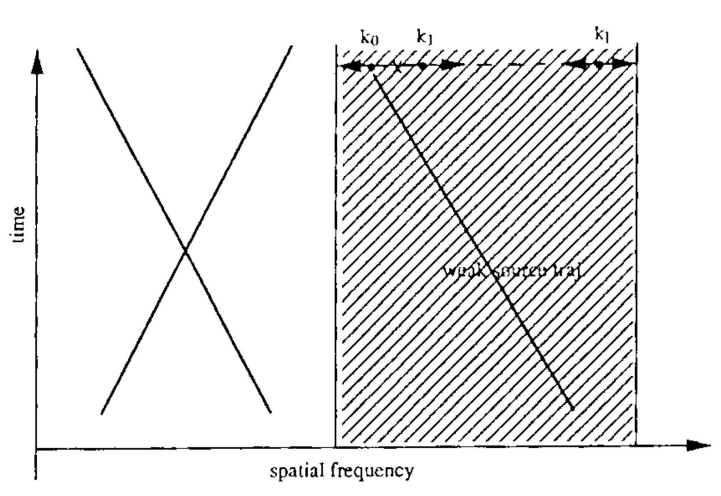

Fig. 8. Spatial sectors.

These equalities lead us to consider the following matrix $\mathcal{H}_{B}$ defined below:

$$
\mathcal{H}_{B}(t)=\left(\begin{array}{cccc}
D_{k_{0}}^{*} Y_{t} & D_{k_{1}}^{*} Y_{t} & \cdots & D_{k_{\ell}}^{*} Y_{t} \\
D_{k_{0}}^{*} \tilde{Y}_{t+1} & D_{k_{1}}^{*} \tilde{Y}_{t+1} & \cdots & D_{k_{\ell}}^{*} \tilde{Y}_{t+1} \\
\vdots & \vdots & & \vdots
\end{array}\right)
$$

then, as previously, the following factorization holds:

$$
\mathcal{H}_{B}(t)=\mathcal{O} \cdot \mathcal{X}_{t}^{\prime}
$$

Thus, according to (62), the practical utilization of SVD for estimating $F_{1}$ (and the $\left\{\dot{k}_{i}\right\}_{i=1}^{s}$ ) is quite classical $[7,24]$ and relies upon $(62)$.

The above methods may be replaced by an equivalent one based on autoregressive (AR) modeling. This last type of method has the advantage of being easily extended to the modeling of time-varying $\left\{\dot{k}_{i, t}\right\}_{i=1}^{s}$ [28].

The proposed method yields satisfying results for the estimation of multiple spatial-frequency rate $\left\{\dot{k}_{i}\right\}_{i=1}^{s}$, even for weak sources [7] or a time-varying source level, allowing us to consider multiple sources TMA.

\section{CONCLUSION}

The spatial-frequency rate has been introduced in TMA. Besides the addition of a new measurement for improving TMA performance, it represents a fundamental change for TMA since the notion of source trajectory replaces the classical one based on instantaneous bearings. Extension of TMA to these new measurements has been presented but the accent has been, overall, put on the statistical performance study. Especially for the special case of a nonmaneuvering observer, analytical calculations have been conducted. In all the cases, the improvements of the TMA performance due to these measurements are important.

Furthermore, it provides a new approach to the difficult problem of multiple sources TMA. 


\section{REFERENCES}

[1] Nardone, S., Lindgren, A., and Gong, K. (1984) Fundamental properties and performance of conventional bearings. Only target motion analysis.

IEEE Transactions on Automatic Control, AC-29, 9

(Sept. 1984), 775-787.

[2] Lindgren, A., and Gong, K. (1978)

Position and velocity estimation via bearing observations. IEEE Transactions on Aerospace and Electronic Systems, AES-14 (July 1978), 564-577.

[3] Burdic, W. S. (1991) Underwater Acoustic System Analysis, (2nd ed.) Englewood Cliffs, NJ: Prentice-Hall, 1991.

[4] Patzewitsch, J. T., Srinath, M. D., and Black, C. I. (1978) Nearfield performance of passive correlation processing sonars.

Journal of the Acoustical Society of America, 64, 5 (Nov. 1978), 1412-1423.

[5] Le Cadre, J. P., and Zugmeyer, O. (1993)

Temporal integration for array processing.

Journal of Acoustical Society of America, 93, 3 (Mar. 1993), 1471-1481.

[6] Le Cadre, J. P., and Zugmeyer, O. (1990)

Intégration temporelle en traitement d'antenne.

Treizième Colloque GRETSI, Juan-les-Pins, Sept. 16-20, 1991, 689-692.

[7] Zugmeyer, O., and Le Cadre, J. P. (1993)

A new approach to the estimation of source motion parameters, Part I.

Signal Processing, 33, 3 (1993), 287-314.

[8] Zugmeyer, O., and Le Cadre, J. P. (1993)

Sur la précision d'estimation du défilement angulaire

d'une source en mouvement.

Traitement du Signal, 10, 1 (1993), 15-28.

[9] Miller, K. S. (1974)

Complex Stochastic Processes. An Introduction to Theory and Applications.

Reading, MA: Addison-Wesley, 1974.

[10] Burg, J. P., Luenberger, D., and Wenger, D. (1982)

Estimation of structured covariance matrices.

Proceedings of the IEEE, 70 (Sept. 1982), 963-974.

[11] Morgera, S. D. (1992)

The role of abstract algebra in structured estimation theory.

IEEE Transactions on Information Theory, 38, 3 (May 1992), 1053-1065.

[12] Le Cadre, J. P., and Lopez, P. (1984)

Estimation d'une matrice interspectrale de structure imposée. Applications.

Traitement du Signal, 1, 1 (1984), 3-17.

[13] Miller, M. I., Furhmann, D. R., O’Sullivan, J. A., and Snyder, D. L. (1991)

Maximum-likelihood methods for Toeplitz covariance estimation and radar imaging.

In S. Haykin (Ed.), Advances in Spectrum Analysis and Array Processing, Vol. II.

Englewood Cliffs, NJ: Prentice-Hall, 1991.

[14] Dahlquist, G., and Bjork, A. (1974)

Numerical Methods.

Englewood Cliffs, NJ: Prentice-Hall, 1974.
[15] Payne, A. N. (1989)

Observability problem for bearings-only tracking.

International Journal of Control, 49, 3 (1989), 761-768.

[16] Nardone, S. C., and Aidala, V. J. (1981)

Observability criteria for bearings-only target motion analysis.

IEEE Transactions on Aerospace and Electronic Systems, AES-17 (Mar. 1981), 161-166.

[17] Weinstein, E. (1982)

Optimal source localization and tracking form passive array measurements.

IEEE Transactions on Acoustics, Speech and Signal Processing, ASSP-30, 1 (Feb. 1982), 69-76.

[18] Chan, Y. T., and Rudnicki, S. W. (1992)

Bearings-only and doppler-bearing tracking using instrumental variable.

IEEE Transactions on Aerospace and Electronic Systems, 28, 4 (Oct. 1992), 1076-1083.

[19] de Vlieger, J. H., and Gmelig Meyling, R. H. (1992)

Maximum likelihood estimation for long range target tracking using passive sonar measurements.

IEEE Transactions on Signal Processing, 40, 5 (May 1992), 1216-1225.

[20] Bar-Shalom, Y., and Fortmann, T. E. (1988)

Tracking and Data Association.

New York: Academic Press, 1988.

[21] Reid, D. B. (1979)

An algorithm for tracking multiple targets.

IEEE Transactions on Automatic Control, AC-24 (Dec. 1979), 843-854.

[22] Ting, P. Y., and Iltis, R. A. (1991)

Multitarget motion analysis in a DSN.

IEEE Transactions on Systems, Man, and Cybernetics, 21, 5 (Sept./Oct. 1991), 1125-1139.

[23] Jauffret, C. G. (1993)

Trajectographie passive, observabilité et prise en compte des fausses alarmes.

Thèse de doctorat de l'université de Toulon et du Var, 18 février 1993.

[24] Kung, S. Y., Arun, K. S., and Bhaskar Rao, D. V. (1983)

State space and singular value decomposition based approximation methods for the harmonic retrieval problem.

Journal of the Optical Society of America, 73, 12 (Dec. 1983), 1789-1811.

[25] Dewilde, P., and Deprettere, E. F. (1988)

Singular value decomposition, an introduction.

In E. F. Deprettere (Ed.), SVD and Signal Processing. Amsterdam: North-Holland, 1988.

[26] Faurre, P. (1976)

Stochastic realization algorithms.

In R. K. Mehra and D. G. Lainiotis (Eds.), Systems

Identification: Advances and Case Studies.

New York: Academic Press, 1976.

[27] Horn, R. A., and Johnson, C. R. (1987)

Matrix Analysis.

New York: Cambridge University Press, 1987.

[28] Le Cadre, J. P., and Zugmeyer, O. (1994)

A new approach to the estimation of source motion parameters, Part II.

Signal Processing, 37, 3 (1994). 

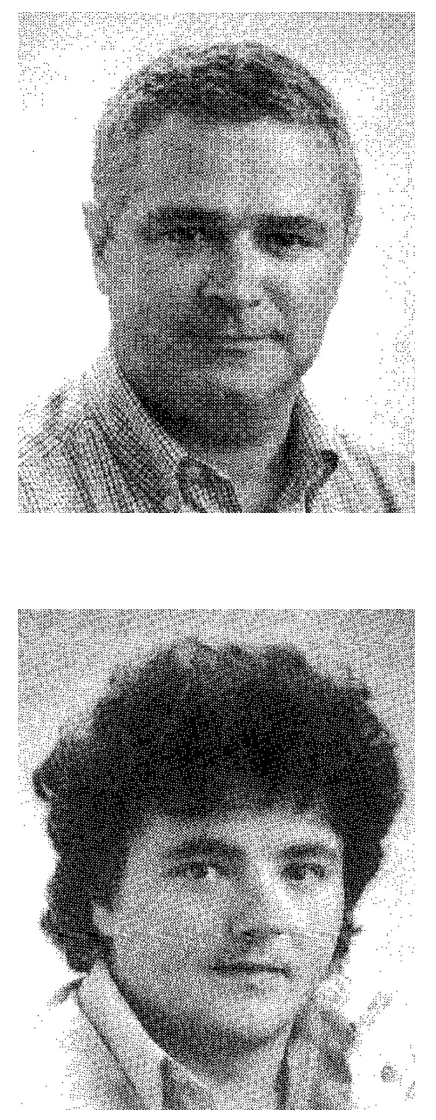

J. P. Le Cadre (M'93) received the M.S. degree in mathematics in 1977, the Doctorat de $3^{-e m e}$ cycle in 1982 and the Doctorat d'Etat in 1987, both from INPG, Grenoble.

From 1980 to 1989, he worked at the GERDSM (Groupe d'Etudes et de Recherche en Detection Sous-Marines), a laboratory of the DCN (Direction des Constructions Navales), mainly on array processing. Since 1989, he has been with IRISA/CNRS, holding a CNRS research position and involved in various projects with DCN. His interests have now moved towards topics like system analysis, detection, data association and operations research.

He received (with O. Zugmeyer) the Signal Processing best paper award in 1993. He is a member of various societies of IEEE.

Olivier Trémois graduated from the École Nationale Supérieure de Physique de Marseille in 1990 and received his Diplôme d'Étude Approfondie in signal processing from the University of Rennes 1 in 1992.

He joined IRISA in 1992 and received the Ph.D. degree in signal processing in June 1995. His research interests are target tracking and target motion analysis.

Since November 1995, he is with Thomson-Sintra ASM Brest working on SONAR projects. 